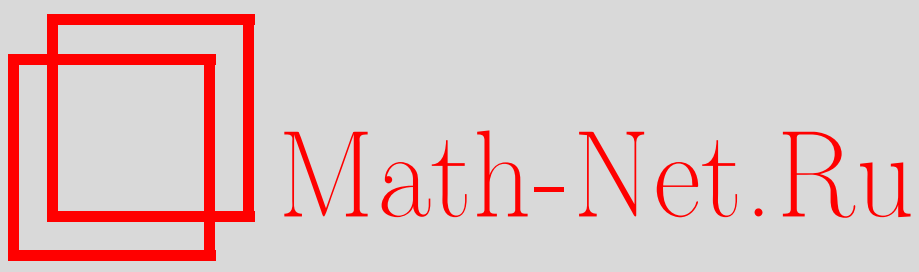

А. В. Рождественский, Об аддитивном когомологическом уравнении и замене времени в линейном потоке на торе с диофантовым вектором частот, Матем. сб., 2004, том 195, номер 5, 115-156

DOI: https://doi.org/10.4213/sm824

Использование Общероссийского математического портала Math-Net.Ru подразумевает, что вы прочитали и согласны с пользовательским соглашением

http://www. mathnet.ru/rus/agreement

Параметры загрузки:

IP : 54.166 .219 .16

26 апреля 2023 г., 09:56:43 
УДК 517.9

\section{А.В. Рождественский}

\section{Об аддитивном когомологическом уравнении и замене времени в линейном потоке на торе с диофантовым вектором частот}

Для 1-периодической функции $f$ конечной гладкости и диоффантова вектора $\alpha$ исследуется проблема разрешимости аддитивного когомологического уравнения на торе

$$
w\left(T_{\alpha} x\right)-w(x)=f(x)-\int_{\mathbb{T}^{d}} f(t) d t,
$$

где $T_{\alpha} x=x+\alpha(\bmod 1)-$ сдвиг тора $\mathbb{T}^{d}$ на вектор $\alpha$, а $w$ - неизвестная измеримая функция.

Получены необходимые и достаточные условия сопряженности линейного потока на $(d+1)$-мерном торе репараметризованному потоку

$$
\left\{\begin{array}{l}
\dot{x}=\frac{\alpha}{F(x, y)}, \\
\dot{y}=\frac{1}{F(x, y)},
\end{array}\right.
$$

где $F(x, y)$ - положительная 1-периодическая функция конечной гладкости.

Библиограффия: 26 названий.

\section{Введение}

Пусть $\mathbb{T}^{d}=\mathbb{R}^{d} / \mathbb{Z}^{d}-d$-мерный тор, $d \in \mathbb{N}$. Тор $\mathbb{T}^{d}$ будем представлять как $d$-мерный куб $\mathscr{E}^{d}=[0,1]^{d}$ с отождествленньми противоположньми гранями. Вектор $\alpha=\left(\alpha_{1}, \ldots, \alpha_{d}\right) \in \mathbb{R}^{d}$ назовем существенно иррачиональньц, если числа $1, \alpha_{1}, \ldots, \alpha_{d}$ линейно независимы над $\mathbb{Z}$. Пусть $T_{\alpha}: \mathbb{T}^{d} \rightarrow \mathbb{T}^{d}, T_{\alpha} x=x+\alpha(\bmod 1)$ - преобразование сдвига тора $\mathbb{T}^{d}$ на вектор $\alpha$.

Следующее функциональное уравнение, в котором $w$ - неизвестная измеримая функция, а $f$ - заданная суммируемая на $\mathbb{T}^{d}$ действительнозначная функция,

$$
w\left(T_{\alpha} x\right)-w(x)=f(x)-\int_{\mathscr{E} d} f(t) d t \quad \forall x \in \mathbb{T}^{d}
$$

называется аддитивным когомологическим уравнением. Функция $f$ назьвается тривиальным над $T_{\alpha}$ аддитивнымм коциклом, если уравнение (1) имеет измеримое решение.

Работа вьполнена при поддержке Российского фонда фундаментальных исследований (грант № 02-01-00248) и Программы поддержки ведущих научных школ РФ (грант № НШ$304.2003 .1)$. 
ЗАмЕчАНИЕ. Поясним происхождение терминов "когомологическое уравнение" и “тривиальный коцикл". Пусть $T$ - автоморфизм пространства Лебега $(X, \mu)$, а группа $\mathbb{Z}$ действует на $X$ итерациями автоморфизма $T$ (т.е. действие элемента $k \in \mathbb{Z}$ на $X$ есть автоморфизм $\left.T^{k}\right)$. Пусть $\Gamma(X)$ обозначает группу измеримых функций на $X$ со значениями в абелевой топологической группе $\Gamma$. Koгомологиями динамической системы $(X, \mu, T)$ называется $H^{1}(\mathbb{Z}, \Gamma(X))$ - группа 1 -мерных когомологий группы $\mathbb{Z}$ со значениями в группе $\Gamma(X)$. Элементы $\Gamma(X)$ назовем коциклами. Два коцикла $\varphi_{1}$ и $\varphi_{2}$ называются когомологичнылми, если $h(x) \varphi_{1}(x)=\varphi_{2}(x) h(T x) \forall x$ для некоторого коцикла $h$. Групша $H^{1}(\mathbb{Z}, \Gamma(X))$ изоморфна групше классов эквивалентности когомологичных коциклов (см., например, [1], [2]).

Пусть $\alpha \in \mathbb{R}^{d}$ - сушественно иррациональный вектор. Линейньцм потоком $R_{\alpha, 1}^{t}$ на торе $\mathbb{T}^{d+1}$ назьвается фазовый поток следующей системы дифференциальных уравнений:

$$
\frac{d x}{d t}=\alpha, \quad \frac{d y}{d t}=1,
$$

где $x \in \mathbb{T}^{d}$ и $y \in \mathbb{T}$.

Для заданной непрерывной положительной 1-периодической функции

$$
F: \mathbb{T}^{d+1} \rightarrow \mathbb{R}_{+}, \quad \int_{\mathscr{E} d+1} F(z) d z=1
$$

мы определим линейный поток с измененным временем (или репараметризованный поток) $R_{\alpha, F}^{t}$ как фазовый поток следуюшей системы дифференциальных уравнений на $(d+1)$-мерном торе:

$$
\frac{d x}{d t}=\frac{\alpha}{F(x, y)}, \quad \frac{d y}{d t}=\frac{1}{F(x, y)} .
$$

Пусть $\beta \geqslant 0$. Вектор $\alpha \in \mathbb{R}^{d}$ называется $\beta$-диофантовыц, если сушествует положительная константа $C_{\alpha}$ такая, что

$$
|q|^{d+\beta}\|q \alpha\| \geqslant C_{\alpha}
$$

при всех $q \in \mathbb{Z}^{d}, q \neq 0$.

Изложим план статьи.

В $\S 1$ мы вводим определения строго $\beta$-диофантова вектора, пространства Орлича, изотропного и анизотропного пространств Орлича-Лиувилля и ОрличаВейля (часто используемые в качестве определения классов Орлича-Соболева для дробной гладкости) и другие используемые в тексте статьи определения.

В $\S 2$ приведены необходимые для понимания теорем $\S \S 4-7$ факты о вложениях пространств Орлича-Лиувилля в пространства Орлича, Соболева и Гёльдера.

В $\S 3$ доказываются некоторые арифметические свойства последовательности $\left\{|k|^{-d-\beta}\|\alpha k\|^{-1}\right\}_{k \in \mathbb{Z}^{d} \backslash\{0\}}$ и вытекающие из них свойства соответствующего мультипликатора Фурье.

В $\S 4$ для типичных функций из обобщенных пространств Орлича-Лиувилля (среди которых, в частности, содержатся классы Орлича-Лиувилля и ОрличаВейля) доказьвается расходимость по мере Лебега биркгофовых сумм $\sum_{s=0}^{n-1} f \circ T_{\alpha}^{s}$. 
В $\S 5$ из результатов $\S 4$ выводятся достаточные условия нетривиальности аддитивных коциклов из пространств Орлича-Лиувилля и Орлича-Вейля и доказываются достаточные условия наличия суммируемого решения когомологического уравнения (1) и достаточные условия отсутствия у этого уравнения непрерывного решения.

$\mathrm{B} \S 6$ для функции $F$ из анизотропного пространства Орлича-Лиувилля или Opлича-Вейля устанавливаются условия изоморфности репараметризованного потока $R_{\alpha, F}$ линейному, а также - условия изоморфности так называемого специального потока над сдвигом $T_{\alpha}$ специальному потоку с "постоянной крьшей".

В $\S 7$ устанавливаются достаточные условия непрерывности спектра косого сдвига Анзаи тора $\mathbb{T}^{d} \times S^{1}$ на ортогональном дополнении в $L^{2}\left(\mathbb{T}^{d} \times S^{1}\right)$ к подпространству функций, зависящих только от первых $d$ координат, а также доказываются достаточные условия топологической транзитивности каскада на цилиндре $\mathbb{T}^{d} \times \mathbb{R}$.

\section{§ 1. Основные определения и обозначения}

Для $x, y \in \mathbb{R}^{d}$ обозначим $x y=\sum_{\nu} x_{\nu} y_{\nu},|x|_{2}=\sqrt{x x},|x|=|x|_{\infty}=\max _{\nu}\left|x_{\nu}\right|$, а для $z \in \mathbb{R}$ через $\|z\|$ обозначим расстояние от $z$ до ближайшего целого числа, $\|z\|=\min \{\{z\}, 1-\{z\}\}$.

ОПРедЕЛЕниЕ 1 . Пусть $\alpha \in \mathbb{R}^{d}-\beta$-диоффантов вектор. Будем говорить, что $\alpha-$ строго $\beta$-диофантов вектор, если сушествует бесконечная последовательность $\left\{q_{s}\right\}_{s=1}^{\infty} \subset \mathbb{Z}^{d}, 0<\left|q_{1}\right|<\left|q_{2}\right|<\cdots$, и константа $C^{\alpha}$ такие, что

$$
\left|q_{s}\right|^{d+\beta}\left\|q_{s} \alpha\right\| \leqslant C^{\alpha}
$$

при всех $s \in \mathbb{N}$.

Сушествование при каждом $\beta>0$ строго $\beta$-диофантовых векторов доказано Н.Г. Мощевитиным в работе [3]. В этой работе также показано, что $\beta$-диофантовы векторы образуют всюду плотное в $\mathscr{E} d$ множество типа $G_{\delta}$. Заметим также, что в силу теоремы Дирихле о совместных приближениях (см. [4; гл. 2, 1 1]) 0-диофантовы векторы автоматически являются строго 0-диофантовыми. В теории диофантовых приближений 0 -диофантовы векторы обычно называются плохо приближаемиции.

Будем говорить, что типичнъий элемент метрического пространства $X$ обладает заданным свойством А, если множество элементов $X$, обладающих свойством А, содержит в себе всюду плотное в $X$ подмножество типа $G_{\delta}$.

Выпуклую функцию $\psi:[0,+\infty) \rightarrow[0,+\infty)$ назовем функцией Юнга, если $\psi(0)=0$ и $\psi \neq \equiv 0$. Для функции Юнга $\psi$ определим класс Орлича $\psi(L)\left(\mathbb{T}^{d}\right)$ :

$$
\begin{aligned}
\psi(L)\left(\mathbb{T}^{d}\right)=\{f: & \mathbb{T}^{d} \rightarrow \mathbb{R}: f-\text { измеримая функция, } \\
& \left.\int_{\mathscr{E} d} \psi(\lambda|f(x)|) d x<\infty \text { для некоторого } \lambda>0\right\} .
\end{aligned}
$$

Нетрудно видеть, что $\psi_{1}(L)\left(\mathbb{T}^{d}\right) \subset \psi_{2}(L)\left(\mathbb{T}^{d}\right)$, если функции Юнга $\psi_{1}$ и $\psi_{2}$ 
удовлетворяют соотношению $\psi_{2}(y)=o\left(\psi_{1}(y)\right), y \rightarrow+\infty$, и что класс $\psi(L)\left(\mathbb{T}^{d}\right)$ совпадает с пространством $L^{p}\left(\mathbb{T}^{d}\right)$, если $\psi(y)=y^{p}, 1 \leqslant p<\infty .1$

Говорят, что функция Юнга $\psi$ удовлетворяет $\Delta_{2}$-условию, если сушествуют $K>0$ и $y_{0}$ такие, что $\psi(2 y) \leqslant K \psi(y)$ при $y \geqslant y_{0}$. Отметим, что если $\psi$ удовлетворяет $\Delta_{2}$-условию, то требование

$$
\int_{\mathscr{E} d} \psi(\lambda|f(x)|) d x<\infty \quad \text { для некоторого } \lambda>0
$$

равносильно требованию $\int_{\mathscr{E} d} \psi(|f(x)|) d x<\infty$. В этом случае факторпространство $\psi(L)\left(\mathbb{T}^{d}\right)$ по подпространству равных нулю почти всюду функций является банаховым пространством относительно нормы Люксембурга

$$
\|f\|_{\psi(L)}=\inf \left\{\lambda>0: \int_{\mathscr{E} d} \psi\left(\frac{|f(x)|}{\lambda}\right) d x \leqslant 1\right\} .
$$

В случае, когда $\psi(y)=y^{p}, 1 \leqslant p<\infty$, норма Люксембурга эквивалентна стандартной $L^{p}$-норме.

Для $\delta>0$ будем обозначать через $L \log _{+}^{\delta} L\left(\mathbb{T}^{d}\right)$ пространство Орлича, соответствуюшее функции Юнга $\psi(y)=y \ln ^{\delta}(y+e)$.

В теоремах $\S \S 4-7$ на функции Юнга мы будем налагать следуюшее условие регулярности, сфформулированное в работе И. Стейна [5]. Обозначим

$$
\Psi=\{\psi(\cdot) \text { - функция Юнга : } \psi(\sqrt{y}) \text { - вогнутая на }[0,+\infty) \text { функция }\} .
$$

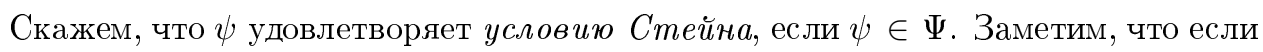
$\psi$ удовлетворяет условию Стейна, то $\psi$ также удовлетворяет $\Delta_{2}$-условию.

Положим $e(y):=\exp (2 \pi i y)$ для $y \in \mathbb{R}$ и $e_{k}(x):=\exp (2 \pi i k x)$ для $k, x \in \mathbb{R}^{d}$. Для суммируемой на $\mathbb{T}^{d}$ функции $f$ через $\widehat{f}_{k}$ или $\widehat{f}(k)$ будем обозначать ее коэффициенты Фурье:

$$
\widehat{f}_{k}=\widehat{f}(k)=\int_{\mathscr{E} d} f(t) e_{-k}(t) d t, \quad k \in \mathbb{Z}^{d} .
$$

Для заданных $r>0$ и $d \in \mathbb{N}$ обозначим через $I_{r}(x)=I_{r}^{d}(x)$ ядро Бесселя-Макдональда - суммируемую на $\mathbb{T}^{d}$ функцию, коэффициенты Фурье которой равны $\widehat{I_{r}}(k)=\left(1+|k|_{2}^{2}\right)^{-r / 2}, k \in \mathbb{Z}^{d}$.

Пусть $f(x) \in L^{1}\left(\mathbb{T}^{d}\right), F(x, y) \in L^{1}\left(\mathbb{T}^{d} \times \mathbb{T}^{l}\right), l \geqslant 0$. (В случае $l=0$ полагаем $F(x, y)=F(x)$.) Тогда сверткой $f$ $u F$ будем называть функцию $f * F: \mathbb{T}^{d+l} \rightarrow \mathbb{R}$,

$$
(f * F)(x, y)=\int_{\mathscr{E} d} f(x-z) F(z, y) d z
$$

\footnotetext{
${ }^{1}$ Мы обозначаем через $L^{p}\left(\mathbb{T}^{d}\right), 1 \leqslant p<\infty$, пространство действительнозначных функций, суммируемых на $\mathscr{E}^{d}$ в степени $p$, а через $L^{\infty}\left(\mathbb{T}^{d}\right)$ - пространство существенно ограниченных действительнозначных функций на $\mathscr{E}^{d}$. Отметим, что факторпространство $L^{p}\left(\mathbb{T}^{d}\right)$, $1 \leqslant p \leqslant \infty$, по подпространству равных нулю почти всюду функций (котороемы также будем обозначать через $\left.L^{p}\left(\mathbb{T}^{d}\right)\right)$ есть банахово пространство относительно стандартной $L^{p}$-нормы:
}

$$
\|f\|_{p}= \begin{cases}\left(\int_{\mathscr{E} d}|f(x)|^{p} d x\right)^{1 / p}, & 1 \leqslant p<+\infty \\ \operatorname{ess~sup}_{x \in \mathbb{T}^{d}}|f(x)|, & p=+\infty\end{cases}
$$


ОпРЕДЕЛЕНИЕ 2. Пусть $r>0, \psi$ - функция Юнга. Определим (изотропный) класс Орлича-Лиувилля:

$$
I_{r} \psi(L)\left(\mathbb{T}^{d}\right)=\left\{f(x) \in \psi(L)\left(\mathbb{T}^{d}\right): f(x)=\left(I_{r}^{d} * \tilde{f}\right)(x), \tilde{f} \in \psi(L)\left(\mathbb{T}^{d}\right)\right\},
$$

и анизотропный класс Орлича-Лиувилля:

$$
\begin{aligned}
I_{r, x} \psi(L)\left(\mathbb{T}^{d+1}\right)=\left\{F(x, y) \in \psi(L)\left(\mathbb{T}^{d} \times \mathbb{T}\right):\right. & \\
& \left.F(x, y)=\left(I_{r}^{d} * \widetilde{F}\right)(x, y), \widetilde{F} \in \psi(L)\left(\mathbb{T}^{d+1}\right)\right\} .
\end{aligned}
$$

Обозначим также

$$
I_{r} C\left(\mathbb{T}^{d}\right)=\left\{f(x)=\left(I_{r} * \tilde{f}\right)(x): \tilde{f} \in C\left(\mathbb{T}^{d}\right)\right\} .
$$

Класс Орлича-Лиувилля $I_{r} \psi(L)\left(\mathbb{T}^{d}\right)$ можно рассматривать в качестве определения класса Орлича-Соболева в случае нецелого $r$, так как при $1<p<\infty$ и $r \in \mathbb{N}$ пространство $W^{r} L^{p}\left(\mathbb{T}^{d}\right)$ совпадает с пространством $I_{r} L^{p}\left(\mathbb{T}^{d}\right)$ (см., например, $[6 ;$ гл. $5, \S 3])^{2}$

В случае $d=1$ альтернативой такого определения пространства Соболева для дробной гладкости может служит пространство Орлича-Вейля (которое мы будем также обозначать через $\left.W^{r} \psi(L)(\mathbb{T})\right)$ - класс периодических функций, дробная в смысле Вейля производная которых принадлежит $\psi(L)$, т.е.

$$
W^{r} \psi(L)(\mathbb{T})=\left\{f \in \psi(L)(\mathbb{T}): f(x)=\left(B_{r} * \tilde{f}\right)(x), \tilde{f} \in \psi(L)(\mathbb{T})\right\},
$$

где $B_{r}(x) \sim 1+\sum_{k \neq 0}(2 \pi)^{-1} \exp \left(-\frac{\pi}{2} i r \operatorname{sign} k\right)|k|^{-r} e_{k}(x)-$ ядро Бернулли.

В случае нецелого $r$ анизотропный класс Орлича-Лиувилля $I_{r, x} \psi(L)\left(\mathbb{T}^{d+1}\right)$ обычно называют анизотропным классом Орлича-Соболева. (Для натурального $r$ анизотропньй класс Орлича-Соболева $W_{x}^{r} \psi(L)\left(\mathbb{T}^{d+1}\right)$ мы определяем как множество функций из $\psi(L)\left(\mathbb{T}^{d+1}\right)$, для которых обобшенные производные порядков $l=\left(l_{1}, \ldots, l_{d}, 0\right), \quad \sum_{1 \leqslant s \leqslant d}\left|l_{s}\right|=r$, также принадлежат пространству $\psi(L)\left(\mathbb{T}^{d+1}\right)$.)

Определим также анизотропњый класс Орлича-Вейля

$$
W_{x}^{r} \psi(L)\left(\mathbb{T}^{2}\right)=\left\{F \in \psi(L)\left(\mathbb{T}^{2}\right): F(x, y)=\left(B_{r} * \widetilde{F}\right)(x, y), \widetilde{F} \in \psi(L)\left(\mathbb{T}^{2}\right)\right\} .
$$

Ясно, что когда $r$ натуральное, a $d=1$, класс $W_{x}^{r} \psi(L)\left(\mathbb{T}^{2}\right)$ совпадает с введенньм вьше анизотропньм пространством Орлича-Соболева.

Через $\mathscr{T}^{d}$ обозначим пространство действительнозначных тригонометрических полиномов от $d$ переменных,

$$
\mathscr{T}^{d}=\left\{\operatorname{Re}\left(\sum_{k \in \mathbb{Z}^{d},|k| \leqslant N} c_{k} e_{k}(x)\right): c_{k} \in \mathbb{C}, N \in \mathbb{N}\right\},
$$

а через $\mathscr{T}_{0}^{d}$ - подпространство $\mathscr{T}^{d}$, состоящее из полиномов с нулевым средним.

\footnotetext{
${ }^{2}$ Изотропный класс Орлича-Соболева $W^{r} \psi(L)\left(\mathbb{T}^{d}\right)$ есть множество функций из пространства $\psi(L)\left(\mathbb{T}^{d}\right)$, для которых обобщенные производные порядков $l=\left(l_{1}, \ldots, l_{d}\right) \in \mathbb{Z}_{+}^{d}$, $\sum_{1 \leqslant s \leqslant d}\left|l_{s}\right|=r$, принадлежат классу $\psi(L)\left(\mathbb{T}^{d}\right)$.
} 
Наконец, обозначим через $L^{0}\left(\mathbb{T}^{d}\right)$ пространство действительнозначных измеримых на $\mathscr{E} d$ функций.

В $\S \S 4-7$ мы будем использовать следующие метрики: $L^{0}$-метрику

$$
\rho_{0}(f, g)=\int_{\mathscr{E} d} \frac{|f(x)-g(x)|}{1+|f(x)-g(x)|} d x
$$

(напомним, что сходимость в $L^{0}\left(\mathbb{T}^{d}\right)$ эквивалентна сходимости по мере); "равномерную" метрику

$$
\rho_{C}(f, g)=\max _{x \in \mathscr{E}^{d}}|f(x)-g(x)|
$$

(для непрерывных на $\mathbb{T}^{d}$ функций); “среднеквадратичную” метрику

$$
\rho_{2}(f, g)=\left(\int_{\mathscr{E} d}|f(x)-g(x)|^{2} d x\right)^{1 / 2}
$$

(для подмножеств пространства $L^{2}\left(\mathbb{T}^{d}\right)$ ).

Символ "0" в качестве нижнего индекса в обозначении для функционального пространства будет означать пересечение этого пространства с классом интегрируемых функций с нулевым средним.

ОПреДЕлЕниЕ 3 . Пусть $\alpha \in \mathbb{R}^{d}$ - существенно иррациональный вектор. Последовательность натуральных чисел $\left\{m_{j}\right\}_{j=1}^{\infty}$ назовем $\alpha$-допустимой, если последовательность векторов $v_{j}=\left(m_{j} \alpha_{1}(\bmod 1), \ldots, m_{j} \alpha_{d}(\bmod 1)\right), j=1,2, \ldots$, имеет предел.

\section{§2. Некоторые сведения о вложении пространств Орлича-Лиувилля}

Отметим некоторые факты о вложениях пространств Орлича-Лиувилля в пространства Орлича, Орлича-Соболева и Гёльдера.

В этом параграфе условимся не различать непрерьвную функцию и функцию, которую можно сделать непрерывной, изменив ее на множестве меры нуль.

1. Кроме отмеченного в $\S 1$ совпадения при натуральном $r$ и $p \in(1,+\infty)$ пространств $I_{r} L^{p}$ и $W^{r} L^{p}$ справедливы включения

$$
W^{2 m} L^{1}\left(\mathbb{T}^{d}\right) \subset I_{2 m} L^{1}\left(\mathbb{T}^{d}\right), \quad W^{2 m} L^{\infty}\left(\mathbb{T}^{d}\right) \subset I_{2 m} L^{\infty}\left(\mathbb{T}^{d}\right),
$$

а также равенства

$$
W^{2 m} L^{1}(\mathbb{T})=I_{2 m} L^{1}(\mathbb{T}), \quad W^{2 m} L^{\infty}(\mathbb{T})=I_{2 m} L^{\infty}(\mathbb{T})
$$

при всех $m \in \mathbb{N}($ см. $[6 ;$ гл. $5, \S 6])$.

2. Элементарньми вычислениями убеждаемся в том, что $\sum_{k}\left(1+|k|_{2}^{2}\right)^{-r / 2}<\infty$ при $r>d$ и, значит, при таких $r$ функция $I_{r}(x)$ непрерывна на $\mathbb{T}^{d}$. Более того, если $0<\varepsilon<r-d$, то $I_{r} * f=I_{r-d-\varepsilon} * I_{d+\varepsilon} * f \forall f \in L^{1}\left(\mathbb{T}^{d}\right)$ и, следовательно, $I_{r} L^{1}\left(\mathbb{T}^{d}\right) \subset I_{r-d-\varepsilon} C\left(\mathbb{T}^{d}\right)$.

Более подробно исследуем "крайний" случай $r=d$. Известно, что функция $I_{d}(x)$ вне точки $x=0$ бесконечно дифференцируема, а в точке $x=0$ имеет асимптотику $I_{d}(x) \sim C \ln (1 /|x|), x \rightarrow 0, C>0$ (см. [6; гл. 5, §6]). Значит, $I_{d} \in \exp (L)\left(\mathbb{T}^{d}\right)$. 
Пусть $f \in L^{1}\left(\mathbb{T}^{d}\right),\|f\|_{1}=1$. Из интегрального неравенства Йенсена [7; гл. $\left.1, \S 10\right]$ имеем $(\lambda>0)$

$$
\begin{aligned}
\int_{\mathscr{E} d} \exp \left(\left|\lambda I_{d} * f(x)\right|\right) d x=\int_{\mathscr{E} d} \exp \left(\lambda\left|\int_{\mathscr{E} d} I_{d}(x-y) f(y) d y\right|\right) d x \\
\quad \leqslant \int_{\mathscr{E} d} \exp \left(\int_{\mathscr{E} d} \lambda\left|I_{d}(x-y)\right||f(y)| d y\right) d x \\
\quad \leqslant \int_{\mathscr{E} d} \int_{\mathscr{E} d} \exp \left(\lambda\left|I_{d}(x-y)\right|\right)|f(y)| d y d x \\
\quad=\int_{\mathscr{E} d} \exp \left(\lambda\left|I_{d}(x)\right|\right) d x \int_{\mathscr{E} d}|f(y)| d y=\int_{\mathscr{E} d} \exp \left(\lambda\left|I_{d}(x)\right|\right) d x
\end{aligned}
$$

следовательно, $\left\|I_{d} * f\right\|_{\exp (L)} \leqslant\left\|I_{d}\right\|_{\exp (L)}$, а из неравенства Гёльдера (см. [8; гл. $2, \S 9])$ имеем $\left\|I_{d} * f\right\|_{\infty} \leqslant\left\|I_{d}\right\|_{\exp (L)}\|f\|_{L} \log _{+} L$, если $f \in L \log _{+} L\left(\mathbb{T}^{d}\right)$. Из неравенства Гёльдера также следует, что $I_{d} * f \in C\left(\mathbb{T}^{d}\right)$ для любой $f \in L \log _{+} L\left(\mathbb{T}^{d}\right)$.

Итак, оператор $f \mapsto I_{d} * f$ непрерывно действует из $L^{1}\left(\mathbb{T}^{d}\right)$ в $\exp (L)\left(\mathbb{T}^{d}\right)$ и из $L \log _{+} L\left(\mathbb{T}^{d}\right)$ в $L^{\infty}\left(\mathbb{T}^{d}\right)$. Следовательно, по теореме интерполяции для операторов, действуюших в пространствах Орлича (см., например, [9]), заключаем, что если $\eta:[0, \infty) \rightarrow[0, \infty)$ - вогнутая функция, $\eta(0)=0$, а выпуклые функции $\varphi$ и $\psi$ определены по формулам ${ }^{3}$

$$
\varphi^{-1}(y)=y \eta\left(\frac{1}{\ln (1+y)}\right), \quad \psi^{-1}(y)=\ln (1+y) \eta\left(\frac{1}{\ln (1+y)}\right)
$$

то оператор $f \mapsto I_{d} * f$ непрерывно действует из $\varphi(L)\left(\mathbb{T}^{d}\right)$ в $\psi(L)\left(\mathbb{T}^{d}\right)$. В частности, полагая $\eta(y)=y^{\delta}, 0<\delta<1$, заключаем, что оператор $f \mapsto I_{d} * f$ непрерывно действует из $L \log _{+}^{\delta} L\left(\mathbb{T}^{d}\right)$ в $\exp \left(L^{1 /(1-\delta)}\right)\left(\mathbb{T}^{d}\right)$. Итак, мы доказали справедливость вложения $I_{d} L \log _{+}^{\delta} L\left(\mathbb{T}^{d}\right) \subset \exp \left(L^{1 /(1-\delta)}\right)\left(\mathbb{T}^{d}\right), 0<\delta<1$.

Отметим, что пространство $I_{d} L \log _{+}^{\delta} L\left(\mathbb{T}^{d}\right), 0<\delta<1$, содержит функцию $f$, которая бесконечно дифференцируема на $\mathbb{T}^{d}$ всюду, кроме точки $x=0$, а в точке $x=0$ имеет асимптотику

$$
f(x) \sim C \ln ^{1-\delta} \frac{1}{|x|} \ln ^{-2}\left(1+\ln \frac{1}{|x|}\right), \quad C>0
$$

(см. [10]). Из результатов $[7 ;$ гл. $5, \S 2]$ следует, что в случае $d=1$ в качестве такой функции можно взять функцию, ряд Фурье которой есть

$$
\sum_{k=2}^{\infty} \frac{\ln ^{-\delta} k \ln ^{-2}(1+\ln k)}{\sqrt{1+k^{2}}} \cos 2 \pi k x .
$$

3. Нетрудно видеть, что $I_{r} C\left(\mathbb{T}^{d}\right) \subset I_{r} \psi_{1}(L)\left(\mathbb{T}^{d}\right) \subset I_{r} \psi_{2}(L)\left(\mathbb{T}^{d}\right)$, если $\psi_{2}(y)=$ $o\left(\psi_{1}(y)\right)$ при $y \rightarrow \infty$ и $I_{r_{1}} \psi(L)\left(\mathbb{T}^{d}\right) \subset I_{r_{2}} \psi(L)\left(\mathbb{T}^{d}\right)$, если $r_{1} \geqslant r_{2}$. При натуральном $r$ имеем включения $C^{r}\left(\mathbb{T}^{d}\right) \subset W^{r} L^{p}\left(\mathbb{T}^{d}\right), W^{r} L^{p}\left(\mathbb{T}^{d}\right)=I_{r} L^{p} \subset I_{r} L \log _{+}^{\delta}\left(\mathbb{T}^{d}\right)$

\footnotetext{
${ }^{3}$ Здесь $\varphi^{-1}$ и $\psi^{-1}-$ функции, обратные к $\varphi$ и $\psi$ соответственно.
} 
(при $1<p<\infty$ и $\delta>0) .{ }^{4}$ Для нецелых $r$ справедливо включение $I_{r} C\left(\mathbb{T}^{d}\right) \subset$ $C^{r}\left(\mathbb{T}^{d}\right)$ (см. [11; гл. $\left.\left.9, \S 1\right]\right)$. Заметим также, что для всех $r>0$ и $\varepsilon>0$ справедливо включение $C^{r+\varepsilon}\left(\mathbb{T}^{d}\right) \subset I_{r} C\left(\mathbb{T}^{d}\right)$.

4. Ситуацию наличия особенностей у некоторых функций из класса $I_{d} L^{1}\left(\mathbb{T}^{d}\right)$ можно "исправить", заменив ядро Бесселя-Макдональда $I_{d}$ на обобшенное ядро $I_{d}^{\theta}$ со специально подобранным множителем $\theta$. Именно введем в рассмотрение множитель Харди-Литтлвуда $\chi(k)=\exp \left(2 \pi i|k|_{2} \ln \left(1+|k|_{2}\right)\right), k \in \mathbb{Z}^{d}$. В [10] доказано, что если $r>d / 2$, то ядро $I_{r}^{\chi}(x)$ - непрерывная функция. И значит, из очевидных соотношений $I_{d}^{\chi} * f=I_{d / 2-\varepsilon} * I_{d / 2+\varepsilon}^{\chi} * f \forall f \in L^{1}\left(\mathbb{T}^{d}\right)$ заключаем, что $I_{d}^{\chi} L^{1}\left(\mathbb{T}^{d}\right) \subset I_{d / 2-\varepsilon} C\left(\mathbb{T}^{d}\right), \varepsilon \in(0, d / 2)$.

\section{§3. Вспомогательные леммы}

Для $j \in \mathbb{Z}_{+}$положим $\Delta_{j}=\left\{k \in \mathbb{Z}^{d}: 2^{j} \leqslant|k|_{\infty} \leqslant 2^{j+1}-1\right\}$. Нетрудно сосчитать мощность "кругового слоя" $\Delta_{j}$. Именно card $\Delta_{j}=2^{j d}\left(\left(4-2^{-j}\right)^{d}-\right.$ $\left.\left(2-2^{-j}\right)^{d}\right)$.

Для измеримой функции $\lambda$ на пространстве $X$ с мерой $\mu$ и измеримого подмножества $\Omega \subset X$ обозначим через $\lambda^{*}(s ; \Omega)$ невозрастающую перестановку функции $\lambda(s)$ на множестве $\Omega$ :

$$
\lambda^{*}(s ; \Omega)=\inf \{z>0: \mu\{t \in \Omega:|\lambda(t)|>z\} \leqslant s\} .
$$

В случае, когда $\Omega=X$, будем писать $\lambda^{*}(s)$ вместо $\lambda^{*}(s ; \Omega)$. В пространстве $\mathbb{Z}^{d}$ меру подмножества $\Omega$ определим как количество его элементов.

Для заданного $\beta \geqslant 0$ и вектора $\alpha \in \mathbb{R}^{d}$ положим

$$
\lambda_{\alpha, \beta}(k)=\left(|k|^{d+\beta}\|\alpha k\|\right)^{-1}
$$

при $k \in \mathbb{Z}^{d} \backslash\{0\}$ и $\lambda_{\alpha, \beta}(0)=0$.

Лемма 3.1. Пусть $\alpha \in \mathbb{R}^{d}-\beta$-диофантов вектор. Тогда существует положительная константа $C$ такая, что

$$
\lambda_{\alpha, \beta}^{*}\left(s ; \Delta_{j}\right) \leqslant \frac{C}{s^{1+\beta / d}}
$$

при всех $j \in \mathbb{Z}_{+}$и $s=1, \ldots$, card $\Delta_{j}$.

ДокАЗАТЕЛЬСтво. Определим векторы $l_{j}(s) \in \Delta_{j}, s=1, \ldots, \operatorname{card} \Delta_{j}$, из уравнения $\lambda_{\alpha, \beta}^{*}\left(s ; \Delta_{j}\right)=\left|\lambda_{\alpha, \beta}\left(l_{j}(s)\right)\right|$.

\footnotetext{
${ }^{4}$ Напомним, что пространство Гёльдера $C^{r}\left(\mathbb{T}^{d}\right)$ есть пополнение пространства бесконечно диффференцируемых функций на $\mathbb{T}^{d}$ по норме

$$
\|f\|_{C^{r}}= \begin{cases}\sum_{|j| \leqslant[r]}\left\|D^{j} f\right\|_{C}, & r \in \mathbb{N}, \\ \|f\|_{C^{[r]}}+\sum_{|j|=[r]} \sup _{h}|h|^{-\{r\}}\left\|\Delta_{h} D^{j} f\right\|_{\infty}, & r \notin \mathbb{N} .\end{cases}
$$
}


Докажем справедливость неравенства

$$
\left|l_{j}(s)\right|^{d+\beta}\left\|\alpha l_{j}(s)\right\| \geqslant \frac{C_{\alpha}}{2} \frac{s^{1+\beta / d}}{\left(\left(4^{d}-2^{d}\right) 2^{d}\right)^{1+\beta / d}}
$$

при всех $s=1, \ldots, \operatorname{card} \Delta_{j}$. (Нетрудно видеть, что из (5) следует неравенство (4), в котором $C=2\left(\left(4^{d}-2^{d}\right) 2^{d}\right)^{1+\beta / d} / C_{\alpha}$. $)$

При $s=1, \ldots, 4^{d}-2^{d}$ неравенство (5) непосредственно следует из условия $\beta$-диофантовости вектора $\alpha$.

Для фиксированного $S, 4^{d}-2^{d} \leqslant S \leqslant \operatorname{card} \Delta_{j}$, найдем $t \in \mathbb{Z}_{+}$из соотношений

$$
\left(4^{d}-2^{d}\right) 2^{d t}+1 \leqslant S \leqslant\left(4^{d}-2^{d}\right) 2^{d(t+1)} .
$$

"Круговой слой" $\Pi_{j}:=\left\{x \in \mathbb{R}^{d}: 2^{j} \leqslant|x|_{\infty} \leqslant 2^{j+1}\right\}$ разобьемна $\left(\operatorname{vol} \Pi_{j}\right) / 2^{j d}=$ $4^{d}-2^{d}$ равных кубов $\delta_{\nu}$ с ребрами длины $2^{j}$. Каждый такой куб разобьем на $2^{d t}$ равных кубов $\delta_{\nu}^{\mu}$. Получим в итоге разбиение $\Pi_{j}$ на $\left(4^{d}-2^{d}\right) 2^{d t}$ равных кубов с длиной ребра $2^{j-t}$.

В силу принципа выбора Дирихле хотя бы один из кубов $\delta_{\nu}^{\mu}$ содержит сразу две точки из набора $\left\{l_{j}(s): s=1, \ldots, S\right\}$. Обозначим их через $l^{\prime}$ и $l^{\prime \prime}$. Ясно, что $\left|l^{\prime}-l^{\prime \prime}\right| \leqslant 2^{j-t}$ и $\left|l^{\prime}\right| \geqslant 2^{j},\left|l^{\prime \prime}\right| \geqslant 2^{j}$. Поэтому, вспоминая определение векторов $l_{j}(s)$, получим

$$
\left|l_{j}(S)\right|^{d+\beta}\left\|\alpha l_{j}(S)\right\| \geqslant \max \left\{\left|l^{\prime}\right|^{d+\beta}\left\|\alpha l^{\prime}\right\|,\left|l^{\prime \prime}\right|^{d}\left\|\alpha l^{\prime \prime}\right\|\right\} \geqslant 2^{j(d+\beta)} \max \left\{\left\|\alpha l^{\prime}\right\|,\left\|\alpha l^{\prime \prime}\right\|\right\} .
$$

Используя свойства $\|x+y\| \leqslant\|x\|+\|y\|$ и $\|-x\|=\|x\|$, имеем неравенство $\max \left\{\left\|\alpha l^{\prime}\right\|,\left\|\alpha l^{\prime \prime}\right\|\right\} \geqslant\left\|\alpha\left(l^{\prime}-l^{\prime \prime}\right)\right\| / 2$. Поэтому

$$
\begin{aligned}
\left|l_{j}(S)\right|^{d+\beta}\left\|\alpha l_{j}(S)\right\| & \geqslant 2^{j(d+\beta)} \frac{\left\|\alpha\left(l^{\prime}-l^{\prime \prime}\right)\right\|}{2}=\frac{2^{j(d+\beta)}}{\left|l^{\prime}-l^{\prime \prime}\right|^{d+\beta}} \frac{\left|l^{\prime}-l^{\prime \prime}\right|^{d+\beta}\left\|\alpha\left(l^{\prime}-l^{\prime \prime}\right)\right\|}{2} \\
& \geqslant \frac{2^{j(d+\beta)}}{\left|l^{\prime}-l^{\prime \prime}\right|^{d+\beta}} \frac{C_{\alpha}}{2} .
\end{aligned}
$$

Воспользуемся теперь неравенством $\left|l^{\prime}-l^{\prime \prime}\right| \leqslant 2^{j-t}$ и оценками (6), вследствие которых $2^{t} \geqslant S^{1 / d} /\left(\left(4^{d}-2^{d}\right)^{1 / d} 2\right)$. Итак, имеем

$$
\frac{2^{j(d+\beta)}}{\left|l^{\prime}-l^{\prime \prime}\right|^{d+\beta}} \geqslant \frac{2^{j(d+\beta)}}{2^{(j-t)(d+\beta)}}=2^{t(d+\beta)} \geqslant \frac{s^{1+\beta / d}}{\left(4^{d}-2^{d}\right)^{1+\beta / d} 2^{d+\beta}},
$$

а следовательно, справедливо (5), что и требовалось.

ЛЕмма 3.2. Предположим, что множество $D \subset \mathbb{Z}^{d}$ лакунарно в следующем смысле: существует номер $j_{0} \in \mathbb{N}$ такой, что $D \cap \Delta_{j}=\varnothing$ при $j<j_{0} u$ $\operatorname{card}\left(D \cap \Delta_{j}\right)=1$ при $j \geqslant j_{0}$. Тогда

1) существуют положительные константы $c^{1}, c^{2}$ такие, что

$$
\sqrt{\sum_{k \in D}\left|\widehat{g}_{k}\right|^{2}} \leqslant c^{1} \int_{\mathscr{E} d}|g(x)| \ln ^{1 / 2}(e+|g(x)|) d x+c^{2}
$$

для всякой функции $g \in L \log _{+}^{1 / 2} L\left(\mathbb{T}^{d}\right)$; 
2) найдется положительная константа $c^{3}$ такая, что для любой последовательности чисел $\left\{\widehat{g}_{k}\right\}_{k \in D}$, для которой $\sum_{k \in D}\left|\widehat{g}_{k}\right|^{2}<\infty$, существует функиия $g_{D} \in \bigcap_{2 \leqslant q<\infty} L^{q}\left(\mathbb{T}^{d}\right)$ такая, что $\widehat{g}_{D}(k)=\widehat{g}_{k}$, если $k \in D, \widehat{g}_{D}(k)=0$, если $k \notin D, u$

$$
\left\|g_{D}\right\|_{q} \leqslant c^{3} \sqrt{q} \sqrt{\sum_{k \in D}\left|\widehat{g}_{k}\right|^{2}}, \quad q \in[2,+\infty) .
$$

ДоКАЗАТЕЛЬСТво. УПорядочим элементы множества $D$ следующим способом: $D=\left\{d_{m}\right\}_{m=1}^{\infty}$, где $\left\{d_{m}\right\}=D \cap \Delta_{j_{0}+m-1}$. Представим $D$ в виде объединения трех попарно не пересекающихся множеств: $D=D^{0} \cup D^{1} \cup D^{2}, D^{\nu}=\left\{d_{l} \in D: l \equiv \nu\right.$ $(\bmod 3), l \in \mathbb{N}\}$. Элементы множества $D^{\nu}$ упорядочим в порядке возрастания их норм и обозначим через $d_{m}^{(\nu)}, m=1,2, \ldots$. Нетрудно убедиться, что

$$
\left|d_{m+1}^{(\nu)}\right| \geqslant 4\left|d_{m}^{(\nu)}\right|
$$

для любого $m \in \mathbb{N}$.

Пусть $r_{m}:[0,1] \rightarrow \mathbb{R}, m=1,2, \ldots$, обозначает $m$-ю функцию Радемахера:

$$
r_{m}(t)= \begin{cases}1, \quad t \in\left(\frac{i-1}{2^{m}}, \frac{i}{2^{m}}\right), & i \text { нечетное } \\ -1, \quad t \in\left(\frac{i-1}{2^{m}}, \frac{i}{2^{m}}\right), & i \text { четное. }\end{cases}
$$

Зафиксируем произвольный тригонометрический полином $G$, удовлетворяющий условию $\sum_{k \in D}\left|\widehat{G}_{k}\right|^{2} \neq 0$. (В случае, когда $\sum_{k \in D}\left|\widehat{G}_{k}\right|^{2}=0$, неравенства (7) и (8) тривиальны.)

Для $\nu \in\{0,1,2\}$ положим

$$
\tau^{\nu}(x)= \begin{cases}\sum_{k \in D^{\nu}} \widehat{G}_{k} e_{k}(x)\left(\sum_{k \in D^{\nu}}\left|\widehat{G}_{k}\right|^{2}\right)^{-1 / 2}, & \text { если } \sum_{k \in D^{\nu}}\left|\widehat{G}_{k}\right|^{2} \neq 0, \\ 0, & \text { если } \sum_{k \in D^{\nu}}\left|\widehat{G}_{k}\right|^{2}=0 .\end{cases}
$$

Ясно, что $\int_{\mathscr{E} d} \tau^{\nu}(x) \bar{G}(x) d x=\sqrt{\sum_{k \in D^{\nu}}\left|\widehat{G}_{k}\right|^{2}}$. Обозначим через $N$ степень полинома $G$. Рассмотрим произведение Рисса

$$
R_{t}^{\nu}(x)=\prod_{k \in D^{\nu},|k| \leqslant N}\left(1+r_{|k|}(t) \cos \pi k x\right)=1+\sum_{\nu} \gamma_{\nu}(t) \cos \pi \nu x
$$

Легко видеть, что $R_{t}(x) \geqslant 0, \int_{\mathscr{E} d} R_{t}(x) d x=1$ и в силу $(11) \gamma_{d_{m}^{(\nu)}}(t)=r_{\left|d_{m}^{(\nu)}\right|}(t)$. Положим

$$
S_{t}^{\nu}(x)=\sum_{k \in D^{\nu}}{\widehat{\tau^{\nu}}}_{k} r_{|k|}(t) e_{k}(x)
$$


Тогда при всех $t \in[0,1], t \notin\left\{i / 2^{m}: i \in \mathbb{Z}, m \in \mathbb{N}\right\}$, справедливы равенства

$$
2 S_{t}^{\nu} * R_{t}^{\nu}(x)=2 \sum_{k \in D^{\nu}} \widehat{\tau^{\nu}}(k) r_{|k|}(t) \frac{r_{|k|}(t)}{2} e_{k}(x)=\tau^{\nu}(x)
$$

Положим $\chi(u)=\exp \left(u^{2} / 16\right)$. Из интегрального неравенства Йенсена (см. [7; гл. $1, \S 10])$, примененного к выпуклой функции $\chi$, имеем:

$$
\begin{aligned}
\chi\left(\frac{\left|\tau^{\nu}(x)\right|}{2}\right)=\chi\left(\left|S_{t}^{\nu} * R_{t}^{\nu}(x)\right|\right) & \leqslant \chi\left(\int_{\mathscr{E} d}\left|S_{t}^{\nu}(x+u)\right| R_{t}^{\nu}(u) d u\right) \\
& \leqslant \int_{\mathscr{E} d} \chi\left(\left|S_{t}^{\nu}(x+u)\right|\right) R_{t}^{\nu}(u) d u .
\end{aligned}
$$

Следовательно, при $t \in[0,1], t \notin\left\{i / 2^{m}: i \in \mathbb{Z}, m \in \mathbb{N}\right\}$,

$$
\begin{aligned}
\int_{\mathscr{E} d} \chi\left(\frac{\left|\tau^{\nu}(x)\right|}{2}\right) d x \leqslant \int_{\mathscr{E} d} \int_{\mathscr{E} d} \chi\left(\left|S_{t}^{\nu}(x+u)\right|\right) R_{t}^{\nu}(u) d x d u \\
=\int_{\mathscr{E} d} R_{t}^{\nu}(u) d u \int_{\mathscr{E} d} \chi\left(\left|S_{t}^{\nu}(x+u)\right|\right) d x=\int_{\mathscr{E} d} \chi\left(\left|S_{t}^{\nu}(x)\right|\right) d x .
\end{aligned}
$$

Аналогично,

$$
\int_{\mathscr{E} d}\left(\frac{\left|\tau^{\nu}(x)\right|}{2}\right)^{q} d x \leqslant \int_{\mathscr{E} d}\left|S_{t}^{\nu}(x)\right|^{q} d x
$$

при всех $q \in[1,+\infty)$ и $t \in[0,1], t \notin\left\{i / 2^{m}: i \in \mathbb{Z}, m \in \mathbb{N}\right\}$.

$\mathrm{B}[7 ;$ гл. $5, \S 8]$ фактически доказано, что если

$$
h(t)=\sum_{l=1}^{\infty} h_{l} r_{l}(t), \quad \sum_{l=1}^{\infty}\left|h_{l}\right|^{2} \leqslant 1
$$

то

$$
\int_{0}^{1} \exp \left(\frac{|h(t)|^{2}}{16}\right) d t \leqslant \frac{4}{4-e}, \quad \int_{0}^{1}|h(t)|^{q} d t \leqslant(2 \sqrt{q})^{q} \quad \forall q \in[2,+\infty) .
$$

Отсюда, полагая $h_{l}:=\widehat{\tau^{\nu}}(k) e_{k}(x)$ при $l=|k|, k \in D^{\nu}$, и $h_{l}:=0$ для других $l \in \mathbb{N}$, заключаем, что

$$
\int_{0}^{1} \chi\left(\left|S_{t}^{\nu}(x)\right|\right) d t \leqslant \frac{4}{4-e}, \quad \int_{0}^{1}\left|S_{t}^{\nu}(x)\right|^{q} d t \leqslant(2 \sqrt{q})^{q} .
$$

Следовательно,

$$
\int_{0}^{1} \int_{\mathscr{E} d} \chi\left(\left|S_{t}^{\nu}(x)\right|\right) d x d t=\int_{\mathscr{E} d} \int_{0}^{1} \chi\left(\left|S_{t}^{\nu}(x)\right|\right) d t d x \leqslant \frac{4}{4-e}
$$

и, значит, существует $t_{0} \in[0,1], t_{0} \notin\left\{i / 2^{m}: i \in \mathbb{Z}, m \in \mathbb{N}\right\}$, такое, что

$$
\int_{\mathscr{E} d} \chi\left(\left|S_{t_{0}}(x)\right|\right) d x \leqslant \frac{4}{4-e}
$$


Из (10) и последнего неравенства немедленно следует оценка

$$
\int_{\mathscr{E} d} \chi\left(\frac{\left|\tau^{\nu}(x)\right|}{2}\right) d x \leqslant \frac{4}{4-e} .
$$

Рассуждая аналогично, из (11) получаем, что

$$
\int_{\mathscr{E} d}\left|\tau^{\nu}(x)\right|^{q} d x \leqslant 4^{q} q^{q / 2}
$$

при всех $q \in[2,+\infty)$.

Из определения множеств $D^{\nu}$ и полиномов $\tau^{\nu}$ заключаем, что

$$
\sum_{k \in D} \widehat{G}_{k} e_{k}(x)=\sum_{\nu=0}^{2} \sqrt{\sum_{k \in D^{\nu}}\left|\widehat{G}_{k}\right|^{2}} \tau^{\nu}(x)
$$

Отсюда и из (13) получаем

$$
\begin{aligned}
\left\|\sum_{k \in D} \widehat{G}_{k} e_{k}(\cdot)\right\|_{q} & \leqslant \sum_{\nu=0}^{2} \sqrt{\sum_{k \in D^{\nu}}\left|\widehat{G}_{k}\right|^{2}}\left\|\tau^{\nu}\right\|_{q} \\
& \leqslant\left(\sum_{\nu=0}^{2} \sqrt{\sum_{k \in D^{\nu}}\left|\widehat{G}_{k}\right|^{2}}\right) 4 \sqrt{q} \leqslant \sqrt{3} \sqrt{\sum_{k \in D}\left|\widehat{G}_{k}\right|^{2}} 4 \sqrt{q}
\end{aligned}
$$

при всех $q \in[2, \infty)$. То есть справедливо неравенство $(8)$, в котором $c^{3}=4 \sqrt{3}$.

Нетрудно проверить, что сушествуют абсолютные положительные постоянные $A$ и $B$ такие, что $u v \leqslant \chi(u / 2)+A v \sqrt{\ln (e+v)}+B$ при всех неотрицательных $u$ и $v$. Пусть $g \in L \log _{+}^{1 / 2} L\left(\mathbb{T}^{d}\right)$. Полагая $G(x)=\sum_{|k| \leqslant N} \widehat{g}_{k} e_{k}(x)$, где $N$ достаточно велико, из (12) заключаем, что

$$
\begin{aligned}
\sqrt{\sum_{k \in D}\left|\widehat{G}_{k}\right|^{2}} & \leqslant \sum_{\nu=0}^{2} \sqrt{\sum_{k \in D^{\nu}}\left|\widehat{G}_{k}\right|^{2}}=\sum_{\nu=0}^{2} \int_{\mathscr{E} d} \tau^{\nu}(x) \bar{g}(x) d x \leqslant \sum_{\nu=0}^{2} \int_{\mathscr{E} d}\left|\tau^{\nu}(x) g(x)\right| d x \\
& \leqslant \sum_{\nu=0}^{2} \int_{\mathscr{E} d} \chi\left(\frac{\left|\tau^{\nu}(x)\right|}{2}\right) d x+3 \int_{\mathscr{E} d}(A|g(x)| \sqrt{\ln (e+|g(x)|)}+B) d x \\
& \leqslant \frac{12}{4-e}+3 A \int_{\mathscr{E} d}|g(x)| \sqrt{\ln (e+|g(x)|)} d x+3 B .
\end{aligned}
$$

Отсюда, устремляя $N$ к бесконечности, получаем неравенство (7), в котором $c^{1}=3 A, c^{2}=3 B+12 /(3(4-e))$.

Введем в рассмотрение первые арифметические средние ряда Фурье $\sum_{k} c_{k} e_{k}(x)$ :

$$
\sigma_{n_{1}, \ldots, n_{d}}(f)(x)=\frac{1}{\left(n_{1}+1\right) \cdots\left(n_{d}+1\right)} \sum_{k_{1}=0}^{n_{1}} \cdots \sum_{k_{d}=0}^{n_{d}} S_{k_{1}, \ldots, k_{d}}(x)
$$

где $S_{k_{1}, \ldots, k_{d}}(x)=\sum_{\left|j_{1}\right| \leqslant k_{1}} \cdots \sum_{\left|j_{d}\right| \leqslant k_{d}} c_{j} e_{j}(x)$ - прямоугольные частичные суммы. 
Известно [7; гл. 17, §1], что если $1<q<+\infty$ и нормы $\left\|\sigma_{n}(f)\right\|_{q}, n \in \mathbb{Z}_{+}^{d}$, равномерно ограничены, то $\sum_{k} c_{k} e_{k}(x)$ есть ряд Фурье функции $f \in L^{q}\left(\mathbb{T}^{d}\right)$ и $\left\|\sigma_{n}(f)-f\right\|_{q} \rightarrow 0$, когда $\min _{j} n_{j} \rightarrow+\infty$.

Из (7) заключаем, что существует функция $\widetilde{g} \in L^{2}\left(\mathbb{T}^{d}\right)$ такая, что $\widehat{\widetilde{g}}(k)=g_{k}$, если $k \in D, \widehat{\widetilde{g}}(k)=0$, если $k \notin D$.

Зафиксируем $n \in \mathbb{Z}_{+}^{d}$. Полином $\sigma_{n}(\widetilde{g}(x))$ можно представить в виде $\sigma_{n}(\widetilde{g}(x))=$ $\sum_{k} \xi_{k} \widehat{g}_{k} e_{k}(x)$, где $0 \leqslant \xi_{k} \leqslant 1$. Ясно также, что $\sigma_{n}(\widetilde{g}(x))=\widetilde{g}_{0}(x)+\widetilde{g}_{1}(x)+\widetilde{g}_{2}(x)$, где $\widetilde{g}^{\nu}(x)=\sum_{k \in D^{\nu}} \xi_{k} \widehat{g}_{k} e_{k}(x)$. Из (13) следует, что $\left\|\widetilde{g}^{\nu}\right\|_{q} \leqslant 4 \sqrt{q}\left\|\widetilde{g}^{\nu}\right\|_{2}$. Поэтому

$$
\begin{aligned}
\left\|\sigma_{n}(\widetilde{g})\right\|_{q} & \leqslant\left\|\widetilde{g}_{0}\right\|_{q}+\left\|\widetilde{g}_{1}\right\|_{q}+\left\|\widetilde{g}_{2}\right\|_{q} \leqslant 4 \sqrt{q}\left(\left\|\widetilde{g}_{0}\right\|_{2}+\left\|\widetilde{g}_{1}\right\|_{2}+\left\|\widetilde{g}_{2}\right\|_{2}\right) \\
& \leqslant 4 \sqrt{q} \sqrt{3}\left\|\sigma_{n}(\widetilde{g})\right\|_{2} \leqslant 4 \sqrt{q} \sqrt{3}\|\widetilde{g}\|_{2} .
\end{aligned}
$$

Итак,

$$
\|\widetilde{g}\|_{q} \leqslant\left\|\widetilde{g}-\sigma_{n}(\widetilde{g})\right\|_{q}+\left\|\sigma_{n}(\widetilde{g})\right\|_{q} \leqslant\left\|\widetilde{g}-\sigma_{n}(\widetilde{g})\right\|_{q}+4 \sqrt{3} \sqrt{q}\|\widetilde{g}\|_{2}
$$

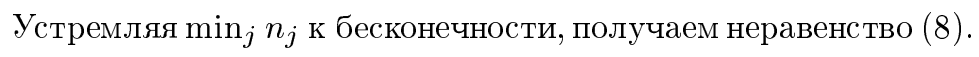

ЗАмЕчАниЕ. В случае $d=1$ доказательство леммы 3.2 содержится в [ $7 ;$ гл. 12 , $\S 7$, гл. $5, \S 8]$.

Для заданной функции $\lambda: \mathbb{Z}^{d} \rightarrow \mathbb{C}$ и натурального $s$ определим (возможно, неоднозначно) векторы $l_{j}(s) \in \Delta_{j}, j=j_{0}, j_{0}+1, \ldots, j_{0}=\min \left\{j: \operatorname{card} \Delta_{j} \geqslant s\right\}$, из уравнения $\lambda^{*}\left(s ; \Delta_{j}\right)=\left|\lambda\left(l_{j}(s)\right)\right|$.

Положим $D_{s}(\lambda)=\left\{l_{j}^{\lambda}(s)\right\}_{j=j_{0}}^{\infty}$. Из определения $\Delta_{j}$ и $D_{s}(\lambda)$ следует, что множества $D_{s}(\lambda), s=1,2, \ldots$, попарно не пересекаются и $\bigcup_{s \in \mathbb{N}} D_{s}(\lambda)=\mathbb{Z}^{d} \backslash\{0\}$.

ЛЕмма 3.3. Предположим, что для функиии $\lambda: \mathbb{Z}^{d} \rightarrow \mathbb{C}, \lambda(0)=0$, найдутся константы $c_{1}>0$ и $\gamma \geqslant 1$ такие, что справедливо неравенство

$$
\lambda^{*}\left(s ; \Delta_{j}\right) \leqslant \frac{c_{1}}{s^{\gamma}}
$$

при всех $j \in \mathbb{N}$ us $=1, \ldots, \operatorname{card} \Delta_{j}$.

Тогда существуют положстельные константы $C_{1}$ и $C_{2}$ такие, что для любой функции $g \in L \log _{+}^{1 / 2} L\left(\mathbb{T}^{d}\right)$ существует функиия $\Lambda g \in \bigcap_{2 \leqslant q<\infty} L^{q}\left(\mathbb{T}^{d}\right)$, коэффичиенты Фурье которой равнь $\lambda(k) \widehat{g}_{k} u$

$$
\|\Lambda g\|_{q} \leqslant q^{\varkappa}\left(C_{1} \int_{\mathscr{E} d}|g(x)| \ln ^{1 / 2}(e+|g(x)|) d x+C_{2}\right), \quad q \in[2,+\infty)
$$

əде $\varkappa=3 / 2$ при $\gamma=1, \quad \varkappa=1 / 2$ при $\gamma>1$.

ДокАЗАТЕльство. Пусть константы $c^{1}, c^{2}$ и $c^{3}$ - из условия леммы 3.2. Введем обозначение: $J(g)=c^{1} \int_{0}^{1}|g(x)| \ln ^{1 / 2}(e+|g(x)|) d x+c^{2}$. 
Пусть $g \in L \log _{+}^{1 / 2} L\left(\mathbb{T}^{d}\right), s \in \mathbb{N}$. Из леммы 3.2 (в которой положим $D=D_{s}(\lambda)$ ) и неравенства (14) следует существование функции $\Lambda_{s} g \in \bigcap_{2 \leqslant q<\infty} L^{q}\left(\mathbb{T}^{d}\right)$ такой, что

$$
\begin{aligned}
& \widehat{\Lambda_{s} g}(k)= \begin{cases}\lambda(k) \widehat{g}_{k}, & k \in D_{s}(\lambda), \\
0, & k \notin D_{s}(\lambda),\end{cases} \\
& \left\|\Lambda_{s} g\right\|_{q} \leqslant c^{3} \sqrt{q} \sqrt{\sum_{k \in D_{s}(\lambda)}|\lambda(k)|^{2}\left|\widehat{g}_{k}\right|^{2}} \leqslant c^{3} \sqrt{q} \frac{c_{1}}{s^{\gamma}} \sqrt{\sum_{k \in D_{s}(\lambda)}\left|\widehat{g}_{k}\right|^{2}} \leqslant c_{4} \frac{\sqrt{q}}{s^{\gamma}} J(g)
\end{aligned}
$$

где $c_{4}=c^{3} c_{1}$.

Обозначим $\Lambda_{s} g(x)=\sum_{k \in D_{s}(\lambda)} \lambda(k) \widehat{g}_{k} e_{k}(x)$.

Пусть $\gamma>1$. Из (16) получим

$$
\sum_{s=1}^{\infty}\left\|\Lambda_{s} g\right\|_{q} \leqslant c_{4} \sqrt{q} J(g) \sum_{s=1}^{\infty} \frac{1}{s^{\gamma}}=c_{5} \sqrt{q} J(g),
$$

где положительная константа $c_{5}$ не зависит от выбора $g$ и $q$. Отсюда следует, что ряд $\sum_{s=1}^{\infty} \Lambda_{s} g$ сходится в $L^{q}\left(\mathbb{T}^{d}\right)$ к функции $\Lambda g$, коэффициенты Фурье которой равны $\lambda(k) \widehat{g}_{k}$, и $\|\Lambda g\|_{q} \leqslant c_{5} \sqrt{q} J(g), q \in[2, \infty)$. Полагая $C_{1}=c_{5} c^{1}, C_{2}=c_{5} c^{2}$, получаем неравенство (15). Пусть теперь $\gamma=1$. Зафиксируем $g \in L \log _{+}^{1 / 2} L\left(\mathbb{T}^{d}\right)$ и определим линейное отображение $T_{g}$ из пространства $l^{\infty}(\mathbb{N})$ в пространство распределений на $\mathbb{T}^{d}$ (т.е. в пространство линейных непрерывных функционалов на $\left.C^{\infty}\left(\mathbb{T}^{d}\right)\right)$ следуюшим способом. Последовательности $u \in l^{\infty}(\mathbb{N})$ поставим в со-

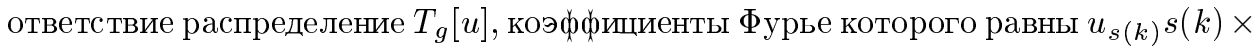
$\lambda(k) \widehat{g}_{k}$, где $s(k)$ однозначно определяется из условия $k \in D_{s}(\lambda)$.

Из (16) и равенства Парсеваля имеем

$$
\left\|T_{g}[u](\cdot)\right\|_{2}^{2}=\sum_{s=1}^{\infty}\left|u_{s}\right|^{2} s^{2}\left\|\Lambda_{s} g(\cdot)\right\|_{2}^{2} \leqslant 2 c_{4}^{2} J^{2}(g)\|u\|_{l^{2}(\mathbb{N})}^{2}
$$

при всех $u \in l^{2}(\mathbb{N})$. Следовательно, линейньй оператор $T_{g}$ непрерьвно действует из $l^{2}(\mathbb{N})$ в $L^{2}\left(\mathbb{T}^{d}\right)$ и для его нормы справедливо неравенство $\left\|T_{g}\right\|_{l^{2} \rightarrow L^{2}} \leqslant$ $\sqrt{2} c_{4} J(g)$.

Пусть $2<q<\infty$. В силу неравенства Минковского и (16) имеем

$$
\left\|T_{g}[u](\cdot)\right\|_{q} \leqslant \sum_{s=1}^{\infty}\left|u_{s}\right| s\left\|\Lambda_{s} g(\cdot)\right\|_{q} \leqslant c_{4} \sqrt{q} J(g)\|u\|_{l^{1}(\mathbb{N})} .
$$

Значит, $T_{g}$ непрерьвно действует из $l^{1}(\mathbb{N})$ в $L^{q}\left(\mathbb{T}^{d}\right)$ и $\left\|T_{g}\right\|_{l^{1} \rightarrow L^{q}} \leqslant c_{4} \sqrt{q} J(g)$.

Пусть $0<\delta<1$. Положим

$$
\frac{1}{p_{1}}=\frac{1-\delta}{1}+\frac{\delta}{2}, \quad \frac{1}{q_{1}}=\frac{1-\delta}{q}+\frac{\delta}{2} .
$$


Применяя теорему Рисса-Торина $[7 ;$ гл. $12, \S 1]$ к оператору $T_{g}$, заключаем, что он непрерывно действует из $l^{p_{1}}(\mathbb{N})$ в $L^{q_{1}}\left(\mathbb{T}^{d}\right)$ и

$$
\begin{aligned}
& \left\|T_{g}\right\|_{l^{p_{1} \rightarrow L^{q_{1}}} \leqslant} \leqslant\left\|T_{g}\right\|_{l^{1} \rightarrow L^{q}}^{1-\delta}\left\|T_{g}\right\|_{l^{2} \rightarrow L^{2}}^{\delta} \\
& \leqslant c_{4} q^{(1-\delta) / 2} J(g)^{1-\delta} 2^{\delta / 2} J(g)^{\delta} \leqslant \sqrt{2} c_{4} q^{(1-\delta) / 2} J(g) .
\end{aligned}
$$

Следовательно, $\left\|T_{g}[u]\right\|_{q_{1}} \leqslant \sqrt{2} c_{4} q^{(1-\delta) / 2} J(g)\|u\|_{l^{p_{1}}}$. В частности, полагая $u=\widehat{u}:=\{1 / s\}_{s=1}^{\infty}$, получаем:

$$
\left\|T_{g}[\widehat{u}]\right\|_{q_{1}} \leqslant \sqrt{2} c_{4} q^{(1-\delta) / 2} \frac{2}{\left(p_{1}-1\right)^{1 / p_{1}}} J(g) .
$$

Из определения $p_{1}$ и $q_{1}$ имеем:

$$
\begin{gathered}
p_{1}-1=\frac{\delta}{2-\delta}, \quad \frac{1}{\left(p_{1}-1\right)^{1 / p_{1}}}=\left(\frac{2}{\delta}-1\right)^{1-\delta / 2}, \\
q_{1}=\frac{2 q}{2(1-\delta)+q \delta}, \quad 2(\delta-1) q_{1}=\left(q_{1} \delta-2\right) q, \quad q=\frac{2(1-\delta)}{2 / q_{1}-\delta} .
\end{gathered}
$$

Поэтому, полагая $\theta=2 / q_{1}$, получаем, что

$$
q^{(1-\delta) / 2} \frac{1}{\left(p_{1}-1\right)^{1 / p_{1}}}=\left(\frac{2(1-\delta)}{\theta-\delta}\right)^{(1-\delta) / 2}\left(\frac{2}{\delta}-1\right)^{1-\delta / 2} .
$$

Выражение в правой части последней формулы обозначим через $F_{\theta}(\delta)$. Покажем, что $\min _{\delta \in(0, \theta)} F_{\theta}(\delta)$ имеет порядок $\theta^{-3 / 2}$ при $\theta \rightarrow 0+$. Действительно, пусть $\bar{\delta}=\bar{\delta}(\theta)$ - точка минимума $F_{\theta}(\delta)$ на $(0, \theta)$. Имеем

$$
F_{\theta}(\bar{\delta}) \sim 2 \sqrt{2} \frac{(\theta-\bar{\delta})^{-1 / 2+\bar{\delta} / 2}}{\bar{\delta}}=2 \sqrt{2} \bar{\delta}^{-3 / 2}\left(\frac{\theta}{\bar{\delta}}-1\right)^{-1 / 2+\bar{\delta} / 2}, \quad \theta \rightarrow 0+.
$$

Ясно, что

$$
F_{\theta}\left(\frac{\theta}{2}\right) \sim 2 \sqrt{2} \theta^{-3 / 2}, \quad \theta \rightarrow 0+.
$$

Если предположить, что $\liminf _{\theta \rightarrow 0+}(\bar{\delta} / \theta)=0$, то, вводя обозначение $\xi=\bar{\delta} / \theta$, получаем

$$
F_{\theta}(\bar{\delta}) \sim 2 \sqrt{2} \theta^{-3 / 2} \xi^{-3 / 2} \frac{(1-\xi)^{-1 / 2+\theta \xi}}{\xi^{-1 / 2+\theta \xi}} \geqslant \theta^{-3 / 2} \xi^{-1}(1-\xi)^{-1 / 2+\theta \xi}, \quad \theta \rightarrow 0+.
$$

Из нашего предположения следует, что $\lim \sup _{\theta \rightarrow 0+} \xi^{-1}(1-\xi)^{-1 / 2+\theta \xi}=+\infty$, что противоречит (18). Полученное противоречие доказывает, что

$$
\min _{\delta \in(0, \theta)} F_{\theta}(\delta) \asymp \theta^{-3 / 2}, \quad \theta \rightarrow 0+.
$$

Из (17) и (19) получаем, что существует положительная константа $c_{6}$ такая, что $\left\|T_{g}[\widehat{u}]\right\|_{q} \leqslant c_{6} q^{3 / 2} J(g)$ при всех $q \in[2, \infty)$. При этом нетрудно видеть, что коэффициенты Фурье функции $T_{g}[\widehat{u}]$ равны $\lambda(k) \widehat{g}_{k}$. Для завершения доказательства осталось положить $C_{1}=c_{6} c^{1}$ и $C_{2}=c_{6} c^{2}$. 
ЛЕмма 3.4. Пусть вектор $\alpha \in \mathbb{R}^{d}$ строго $\beta$-диофантов, и пусть векторь $q_{s}, s=1,2, \ldots$, удовлетворяют неравенству (3). Тогда для каждого $m \in \mathbb{N}$ существует иелое $n,\left|q_{m}\right|^{d+\beta} / C_{\alpha} \leqslant n \leqslant 2\left|q_{m}\right|^{d+\beta} / C_{\alpha}$, такое, ито

$$
\sum_{s=1}^{m} \frac{\sin ^{2} \pi n q_{s} \alpha}{\left|q_{s}\right|^{2(d+\beta)} \sin ^{2} \pi q_{s} \alpha} \geqslant \frac{1}{4 \pi^{2}\left(C^{\alpha}\right)^{2}} m .
$$

ДокАЗАТЕЛЬСТво. Из неравенства (3) следует, что

$$
\sum_{s=1}^{m} \frac{\sin ^{2} \pi n q_{s} \alpha}{\left|q_{s}\right|^{2(d+\beta)} \sin ^{2} \pi q_{s} \alpha} \geqslant \frac{1}{\pi^{2}\left(C^{\alpha}\right)^{2}} \sum_{s=1}^{m} \sin ^{2} \pi n q_{s} \alpha .
$$

Положим $A=\min \left\{l \in \mathbb{Z}: l \geqslant\left|q_{m}\right|^{d+\beta} / C_{\alpha}\right\}$.

Имеем

$$
\sum_{n=A}^{2 A-1} \cos 2 \pi n \alpha q_{s}=\operatorname{Re} \sum_{n=A}^{2 A-1} e^{2 \pi i n \alpha q_{s}}
$$

Следовательно,

$$
\sum_{n=A}^{2 A-1} \cos 2 \pi n \alpha q_{s} \leqslant\left|\sum_{n=A}^{2 A-1} e^{2 \pi i n \alpha q_{s}}\right|=\frac{\left|\sin A \pi q_{s} \alpha\right|}{\left|\sin \pi q_{s} \alpha\right|} \leqslant \frac{1}{2\left\|q_{s} \alpha\right\|} \leqslant \frac{\left|q_{s}\right|^{d+\beta}}{2 C_{\alpha}}
$$

Поэтому

$$
\begin{aligned}
\frac{1}{A} \sum_{n=A}^{2 A-1} & \left(\sum_{s=1}^{m} \sin ^{2} \pi n q_{s} \alpha\right)=\frac{1}{2} \frac{1}{A} \sum_{n=A}^{2 A-1}\left(\sum_{s=1}^{m}\left(1-\cos 2 \pi n \alpha q_{s}\right)\right) \\
= & \frac{1}{2}\left(m-\sum_{s=1}^{m} \frac{1}{A} \sum_{n=A}^{2 A-1} \cos 2 \pi n \alpha q_{s}\right) \geqslant \frac{1}{2}\left(m-\sum_{s=1}^{m} \frac{1}{A} \frac{\left|q_{s}\right|^{d+\beta}}{2 C_{\alpha}}\right) \\
\geqslant & \frac{1}{2}\left(m-\sum_{s=1}^{m} \frac{1}{2}\right)=\frac{m}{4} .
\end{aligned}
$$

ЛЕмма 3.5. Пусть заданы последовательность $\left\{q_{s}\right\}_{s=1}^{\infty} \subset \mathbb{Z}^{d},\left|q_{1}\right|<\left|q_{2}\right|<\cdots$, функиия $\lambda: \mathbb{Z}^{d} \rightarrow \mathbb{C}$ и убвьваюая функиия $\xi: \mathbb{N} \rightarrow \mathbb{R}_{+}$.

Если существует строго убъвающая к нулю функиия $\psi: \mathbb{N} \rightarrow \mathbb{R}_{+}, \psi(2)>$ $\xi(2)$, такая, что справедливо неравенство

$$
\lambda^{*}\left(s ; \Delta_{j}\right) \leqslant \psi(s)
$$

при всех $j \in \mathbb{N}$ us $=1, \ldots$, card $\Delta_{j}$, то для каждого $n \in \mathbb{N}, n \geqslant 4$, существует набор векторов $\left\{q_{1}^{n}, \ldots, q_{n}^{n}\right\} \subset\left\{q_{s}\right\}_{s=1}^{\infty}$ такой, что

$$
\left|\lambda\left(\delta_{1} q_{1}^{n}+\cdots+\delta_{n} q_{n}^{n}\right)\right| \leqslant \xi(n)
$$

всякий раз, когда $\delta_{j} \in\{-1 ; 0 ; 1\}$ u $\sum_{j=1}^{n}\left|\delta_{j}\right|>1$. 
ДоКАЗАТЕЛЬСТво. Переходя в случае необходимости к подпоследовательности, будем полагать, что $\left|q_{s+1}\right| /\left|q_{s}\right| \geqslant 4$ при всех $s \in \mathbb{N}$. Следовательно, для всякого $l \geqslant 2$ и набора $\left\{\delta_{s}\right\}_{s=1}^{l-1}, \delta_{s} \in\{-1 ; 0 ; 1\}$, имеем $\left|\sum_{s=1}^{l-1} \delta_{s} q_{s}\right| \leqslant \sum_{s=1}^{l-1}\left|q_{s}\right| \leqslant\left|q_{l}\right| / 3$. Поэтому $\left| \pm q_{l}+\sum_{s=1}^{l-1} \delta_{s} q_{s}\right| \leqslant 4\left|q_{l}\right| / 3,\left| \pm q_{l}+\sum_{s=1}^{l-1} \delta_{s} q_{s}\right| \geqslant 2\left|q_{l}\right| / 3$. Отсюда заключаем, что если $q_{l} \in \Delta_{j}$, то множество

$$
M_{l}=\left\{q: q=\delta_{l} q_{l}+\sum_{s=1}^{l-1} \delta_{s} q_{s}, \delta_{l} \in\{-1 ; 1\}, \delta_{s} \in\{-1 ; 0 ; 1\}, s=1, \ldots, l-1\right\}
$$

принадлежит объединению $\Delta_{j-1} \cup \Delta_{j} \cup \Delta_{j+1}$, и, следовательно, из условия леммы заключаем, что

$$
\begin{aligned}
\operatorname{card}\left\{q \in M_{l}:|\lambda(q)|>\varepsilon\right\} & \leqslant \operatorname{card}\left\{k \in \Delta_{j-1} \cup \Delta_{j} \cup \Delta_{j+1}:|\lambda(q)|>\varepsilon\right\} \\
& \leqslant 3 \operatorname{card}\{s \in \mathbb{N}: \psi(s)>\varepsilon\}
\end{aligned}
$$

для всякого $\varepsilon>0$. Продолжим функцию $\psi$ до непрерывной строго убываюшей на $[1,+\infty)$ функции, которую обозначим той же буквой.

Зафиксируем $n \geqslant 2$ и положим $L_{n}=\left[3 n \psi^{-1}(\xi(n))\right]$. Введем множества $Q_{1}=$ $\left\{q_{1}, \ldots, q_{L_{n}}\right\}, Q_{2}=\left\{q_{L_{n}+1}, \ldots, q_{2 L_{n}}\right\}, \ldots, Q_{n-1}=\left\{q_{(n-2) L_{n}+1}, \ldots, q_{(n-1) L_{n}}\right\}$, $Q_{n}=\left\{q_{(n-1) L_{n}+1}\right\}$ и докажем, что сушествуют векторы $q_{1}^{n} \in Q_{1}, \ldots, q_{n}^{n} \in Q_{n}$ такие, что справедливо неравенство (20) при всех $\delta_{j} \in\{-1 ; 0 ; 1\}, \sum_{j=1}^{n}\left|\delta_{j}\right|>1$.

Для заданных векторов $w_{2} \in Q_{2}, \ldots, w_{n} \in Q_{n}$ оценим сверху количество векторов $w_{1} \in Q_{1}$, для которых существуют $\delta_{1} \in\{-1 ; 1\}, l \in\{2, \ldots, n\}$ и $\delta_{l} \in\{-1 ; 1\}$ такие, что $\left|\lambda\left(\delta_{l} w_{l}+\delta_{1} w_{1}\right)\right|>\xi(n)$.

Определим $N(l) \in \mathbb{N}$ из равенства $w_{l}=q_{N(l)}$.

Из (21) следует, что

$$
\begin{aligned}
& \sum_{l=2}^{n} \operatorname{card}\left\{w_{1} \in Q_{1}: \exists \delta_{1}, \delta_{l} \in\{-1 ; 1\},\left|\lambda\left(\delta_{1} w_{1}+\delta_{l} w_{l}\right)\right|>\xi(n)\right\} \\
& \quad \leqslant \sum_{l=2}^{n} \operatorname{card}\left\{q \in M_{N(l)}:|\lambda(q)|>\xi(n)\right\} \leqslant(n-1) 3 \psi^{-1}(\xi(n))<L_{n}=\operatorname{card} Q_{1} .
\end{aligned}
$$

Следовательно, сушествует вектор $w_{1}^{0}=\xi_{1}\left(w_{2}, \ldots, w_{n}\right), w_{1}^{0} \in Q_{1}$, такой, что

$$
\left|\lambda\left(\delta_{1} w_{1}^{0}+\delta_{l} w_{l}\right)\right| \leqslant \xi(n)
$$

при всех $l \in\{2, \ldots, n\}$ и $\delta_{1}, \delta_{l} \in\{-1 ; 1\}$.

Далее, если $n \geqslant 3$, то для заданного набора векторов $w_{3} \in Q_{3}, \ldots, w_{n} \in Q_{n}$ оценим сверху количество векторов $w_{2} \in Q_{2}$, для которых существуют $\delta_{1} \in$ $\{-1 ; 0 ; 1\}, \delta_{2} \in\{-1 ; 1\}, l \in\{3, \ldots, n\}$ и $\delta_{l} \in\{-1 ; 1\}$ такие, что $\mid \lambda\left(\delta_{l} w_{l}+\delta_{2} w_{2}+\right.$ $\left.\delta_{1} w_{1}^{0}\left(w_{2}, \ldots, w_{n}\right)\right) \mid>\xi(n)$. Так как

$$
\delta_{l} w_{l}+\delta_{2} w^{\prime}+\delta_{1} \xi_{1}\left(w^{\prime}, w_{3}, \ldots, w_{n}\right) \neq \delta_{l} w_{l}+\delta_{2} w^{\prime \prime}+\delta_{1} \xi_{1}\left(w^{\prime \prime}, w_{3}, \ldots, w_{n}\right),
$$


если $w^{\prime} \neq w^{\prime \prime}, w^{\prime}, w^{\prime \prime} \in Q_{2}$, значит,

$$
\begin{aligned}
& \sum_{l=3}^{n} \operatorname{card}\left\{w_{2} \in Q_{2}: \exists \delta_{2}, \delta_{l} \in\{-1 ; 1\}, \delta_{1} \in\{-1 ; 0 ; 1\},\right. \\
& \left.\left|\lambda\left(\delta_{l} w_{l}+\delta_{2} w_{2}+\delta_{1} \xi_{1}\left(w_{2}, \ldots, w_{n}\right)\right)\right|>\xi(n)\right\} \\
& \leqslant \sum_{l=3}^{n} \operatorname{card}\left\{q \in M_{N(l)}:|\lambda(q)|>\xi(n)\right\} \leqslant(n-2) 3 \psi^{-1}(\xi(n))<L_{n}=\operatorname{card} Q_{2} .
\end{aligned}
$$

Следовательно, сушествует вектор $w_{2}^{0}=\xi_{2}\left(w_{3}, \ldots, w_{n}\right), w_{2}^{0} \in Q_{2}$, такой, что

$$
\left|\lambda\left(\delta_{1} w_{1}^{0}+\delta_{2} w_{2}^{0}+\delta_{l} w_{l}\right)\right| \leqslant \xi(n),
$$

$l \in\{2, \ldots, n\}, \delta_{2}, \delta_{l} \in\{-1 ; 1\}, \delta_{1} \in\{-1 ; 0 ; 1\} .\left(\right.$ Здесь $\left.w_{1}^{0}=\xi_{1}\left(w_{2}^{0}, w_{3}, \ldots, w_{n}\right).\right)$

Пусть $j \in\{3, \ldots, n-1\}, n \geqslant 4$, и уже построены отображения $\xi_{s}: Q_{s+1} \times \cdots \times$ $Q_{n} \rightarrow Q_{s}, s=1, \ldots, j-1$, такие, что при всех $l \in\{j, \ldots, n\}, \delta_{l}, \delta_{j-1} \in\{-1 ; 1\}$, $\delta_{s} \in\{-1 ; 0 ; 1\}, s=1, \ldots, j-2$, справедливо неравенство

$$
\left|\lambda\left(\delta_{l} w_{l}+\delta_{j-1} w_{j-1}^{0}+\delta_{1} w_{1}^{0}+\cdots+\delta_{j-2} w_{j-2}^{0}\right)\right| \leqslant \xi(n),
$$

где $w_{j-1}^{0}=\xi_{j-1}\left(w_{j}, \ldots, w_{n}\right), w_{j-2}^{0}=\xi_{j-2}\left(w_{j-1}^{0}, w_{j}, \ldots, w_{n}\right), \ldots, w_{1}^{0}=\xi_{1}\left(w_{2}^{0}, \ldots\right.$, $\left.w_{j-1}^{0}, w_{j}, \ldots, w_{n}\right)$.

Для $j \geqslant 3, l \in j+1, \ldots, n$ и $w \in Q_{j}$ рассмотрим множества

$$
\begin{aligned}
M^{j, l}(w)=\left\{q: q=\delta_{l} w_{l}+\delta_{j} w+\delta_{1} w_{1}^{0}+\cdots+\delta_{j-1} w_{j-1}^{0},\right. & \\
& \left.\delta_{l}, \delta_{j} \in\{-1 ; 1\}, \delta_{s} \in\{-1 ; 0 ; 1\}, s=1, \ldots, j-1\right\} .
\end{aligned}
$$

Из условия на $q_{s}$ следует, что $M^{j, l}\left(w^{\prime}\right) \cap M^{j, l}\left(w^{\prime \prime}\right)=\varnothing$ при $w^{\prime} \neq w^{\prime \prime}, w^{\prime}, w^{\prime \prime} \in Q_{j}$, и $M^{j, l}(w) \subset M_{N(l)}$ при всех $w \in Q_{j}$. Поэтому

$$
\begin{aligned}
& \sum_{l=j+1}^{n} \operatorname{card}\left\{w_{j} \in Q_{j}: \exists \delta_{j}, \delta_{l} \in\{-1 ; 1\}, \delta_{s} \in\{-1 ; 0 ; 1\},\right. \\
& \left.\left|\lambda\left(\delta_{l} w_{l}+\delta_{j} w_{j}+\sum_{s=1}^{j-1} \delta_{s} w_{s}^{0}\right)\right|>\xi(n)\right\} \\
& \leqslant \sum_{l=j+1}^{n} \operatorname{card}\left\{q \in M_{N(l)}:|\lambda(q)|>\xi(n)\right\} \leqslant(n-j) 3 \psi^{-1}(\xi(n))<L_{n}=\operatorname{card} Q_{j}
\end{aligned}
$$

и, значит, рассуждая как в случае $j=2$, заключаем, что сушествует вектор $w_{j}^{0}=$ $\xi_{j}\left(w_{j+1}, \ldots, w_{n}\right) \in Q_{j}$, удовлетворяюший неравенству

$$
\left|\lambda\left(\delta_{l} w_{l}+\delta_{j} w_{j}^{0}+\delta_{1} w_{1}^{0}+\cdots+\delta_{j-1} w_{j-1}^{0}\right)\right| \leqslant \xi(n)
$$

при всех $l \in\{j+1, \ldots, n\}, \delta_{l}, \delta_{j} \in\{-1 ; 1\}, \delta_{s} \in\{-1 ; 0 ; 1\}, s=1, \ldots, j-1$.

Полагая $q_{n}^{n}=q_{(n-1) L_{n}+1}, q_{n-1}^{n}=\xi_{n-1}\left(q_{n}^{n}\right), q_{n-2}^{n}=\xi_{n-2}\left(q_{n-1}^{n}, q_{n}^{n}\right), \ldots, q_{1}^{n}=$ $\xi_{1}\left(q_{2}^{n}, \ldots, q_{n}^{n}\right)$, из неравенств $(22)-(24)$ заключаем, что набор векторов $\left\{q_{1}^{n}, \ldots, q_{n}^{n}\right\}$ искомый. 


\section{§4. Условия расходимости по мере биркгофовых сумм}

4.1. Введем в рассмотрение следующий класс функций целого аргумента:

$$
\Theta=\left\{\theta: \mathbb{Z}^{d} \rightarrow \mathbb{C}:|\theta(k)| \equiv 1, \theta(0)=0\right\} .
$$

ОПРЕДЕЛЕНИЕ 4.1. Для заданных числа $r \geqslant d$ и функции $\theta \in \Theta$ обозначим через $I_{r}^{\theta}(x)=I_{r}^{d, \theta}(x)$ обобщенное ядро Бесселя-Макдональда - суммируемую на $\mathbb{T}^{d}$ функцию, коэффициенты Фурье которой равны $\widehat{I_{r}^{\theta}}(k)=\theta(k)\left(1+|k|_{2}^{2}\right)^{-r / 2}$.

В случае, когда $d=1$, а $\theta(k)=\exp \left(-\frac{\pi}{2} i r \operatorname{sign} k\right)$, пространство $I_{r}^{\theta} \psi(L)\left(\mathbb{T}^{d}\right)$ совпадает с пространством Орлича-Вейля $W^{r} \psi(L)(\mathbb{T})$.

Введем в рассмотрение обобщенный класс Орлича-Лиувилля:

$$
I_{r}^{\theta} \psi(L)\left(\mathbb{T}^{d}\right)=\left\{f \in \psi(L)\left(\mathbb{T}^{d}\right): f(x)=\int_{\mathscr{E} d} f(t) d t+I_{r}^{\theta} * \widetilde{f}(x), \tilde{f} \in \psi(L)\left(\mathbb{T}^{d}\right)\right\} .
$$

4.2. Исследуем порядок роста $L^{2}$-нормы биркгофовых сумм для функции $I_{d+\beta}^{\theta}$.

Обозначим $K_{n}(x)=\sum_{s=0}^{n-1} I_{d+\beta}^{\theta}\left(T_{\alpha}^{s} x\right), n \in \mathbb{N}$. Нетрудно вычислить коэффициенты Фурье функции $K_{n}(x)$ :

$$
\widehat{K}_{n}(k)=\left(K_{n} * e_{k}(x)\right) e_{-k}(x)=\theta(k) \frac{e_{k}(n \alpha)-1}{\left(1+|k|_{2}^{2}\right)^{(d+\beta) / 2}\left(e_{k}(\alpha)-1\right)} .
$$

Лемма 4.1. Пусть $\alpha \in \mathbb{R}^{d}-\beta$-диофантов вектор, $\theta \in \Theta$. Тогда существуem $C_{3}>0$ makoe, чmo

$$
\left\|\sum_{s=0}^{n-1} I_{d+\beta}^{\theta} \circ T_{\alpha}^{s}\right\|_{2} \leqslant C_{3} \sqrt{\ln (1+n)}
$$

для любого $n \in \mathbb{N}$.

$$
\begin{aligned}
& \text { ДоказАтЕльство. Положим } l=\left[\frac{2 \ln n}{(d+2 \beta) \ln 2}\right]+1 \text {. Имеем } \\
& \qquad\left\|K_{n}\right\|_{2}^{2}=\sum_{q}\left|\widehat{K}_{n}(q)\right|^{2}=\sum_{|q|=1}^{2^{l}-1}\left|\widehat{K}_{n}(q)\right|^{2}+\sum_{|q|=2^{l}}^{\infty}\left|\widehat{K}_{n}(q)\right|^{2}=\sum^{\prime}+\sum^{\prime \prime} .
\end{aligned}
$$

Для оценки сверху $\sum^{\prime}$ используем неравенства

$$
\left|\widehat{K}_{n}(q)\right| \leqslant \frac{1}{|\sin \pi \alpha q||q|^{d+\beta}} \leqslant \lambda_{\alpha, \beta}(q)
$$

и лемму 3.1. Имеем:

$$
\begin{aligned}
\sum^{\prime} & \leqslant \sum_{j=1}^{l-1} \sum_{q \in \Delta_{j}} \lambda_{\alpha, \beta}^{2}(q)=\sum_{j=1}^{l-1} \sum_{s=1}^{\operatorname{card} \Delta_{j}}\left(\lambda_{\alpha, \beta}^{*}\left(s ; \Delta_{j}\right)\right)^{2} \\
& \leqslant C^{2} \sum_{j=1}^{l-1} \sum_{s=1}^{+\infty} \frac{1}{s^{2(1+\beta / d)}} \leqslant C^{2} \frac{\pi^{2}}{6}(l-1)
\end{aligned}
$$


Для оценки сверху $\sum^{\prime \prime}$ используем неравенства

$$
\|n y\| \leqslant n\|y\|, \quad\left|\widehat{K}_{n}(q)\right| \leqslant \frac{\|n q \alpha\|}{|q|^{d+\beta}\|q \alpha\|} .
$$

Имеем:

$$
\begin{aligned}
\sum^{\prime \prime} & \leqslant n^{2} \sum_{|q|=2^{l}}^{\infty} \frac{1}{|q|^{2(d+\beta)}}=n^{2} \sum_{s=2^{l}}^{\infty} \frac{\operatorname{card}\left\{q \in \mathbb{Z}^{d}:|q|=s\right\}}{s^{2(d+\beta)}} \\
& \leqslant C_{1} n^{2} \sum_{s=2^{l}}^{\infty} \frac{1}{s^{d+1+2 \beta}} \leqslant C_{2} \frac{n^{2}}{2^{l(d+2 \beta)}} \leqslant C_{2}
\end{aligned}
$$

где константы $C_{1}$ и $C_{2}$ не зависят от выбора $n$.

Итак, мы доказали, что существует константа $C_{3}$ такая, что

$$
\left\|K_{n}\right\|_{2} \leqslant C_{3} \sqrt{\ln (1+n)}
$$

при всех $n \in \mathbb{N}$, что и требовалось доказать.

Отметим также следуюшее полезное для дальнейшего изложения тождество:

$$
\left(K_{n} * g\right)(x)=\sum_{s=0}^{n-1}\left(I_{d+\beta}^{\theta} T_{\alpha}^{s}\right) * g(x)=\sum_{s=0}^{n-1}\left(I_{d+\beta}^{\theta} * g\right)\left(T_{\alpha}^{s} x\right)
$$

для любого $g \in L^{1}\left(\mathbb{T}^{d}\right)$.

4.3. В работе [12] доказана

ЛЕмма (Пичугов). Пусть $F_{j} \in L^{2}\left(\mathbb{T}^{d}\right), j=1,2, \ldots$ Eсли существует константа $C$ такая, что

$$
\sup _{0<t<1}\left(t\left(F_{j} * g\right)^{*}(t)\right) \leqslant C\|g\|_{1}
$$

для любъх $j \in \mathbb{N}$ u $g \in L^{1}\left(\mathbb{T}^{d}\right)$, то найдутся положительние постоянные $A_{1}$ и $A_{2}$ такие, что для всех $j \in \mathbb{N}$ справедливо неравенство

$$
\left\|F_{j}\right\|_{1} \leqslant A_{1}+A_{2} \ln \left(e+\left\|F_{j}\right\|_{2}\right) .
$$

В работе [13] Пичугов доказал следующее утверждение.

Теорема (Пичугов). Пусть $T_{n}: L^{1}\left(\mathbb{T}^{d}\right) \rightarrow L^{1}\left(\mathbb{T}^{d}\right), n=1,2, \ldots,-$ линейнъе непрерывные операторы, перестановочные с операторами сдвига $\tau_{a} g(x)=$ $g(x+a)$.

Тогда если последовательность $\left\{T_{n} g\right\}_{n=1}^{\infty}$ сходится по мере для каждой $g \in L^{1}\left(\mathbb{T}^{d}\right)$, то найдется постоянная $B>0$ такая, что для всех $n \in \mathbb{N}$, $g \in L^{1}\left(\mathbb{T}^{d}\right)$ выполняется неравенство

$$
\sup _{0<y<1}\left(y\left(T_{n} g\right)^{*}(y)\right) \leqslant B\|g(t)\|_{1} .
$$

В случае $d=1$ следуюшая лемма доказана в работе [14]. Ее доказательство для случая произвольного $d \in \mathbb{N}$ полностью повторяет "одномерное" доказательство (с соответствуюшими изменениями в обозначениях). 
Лемма 4.2. Пусть $\psi \in \Psi, \mathbf{K}_{j} \in L^{2}\left(\mathbb{T}^{d}\right), \mathbf{v}_{j} \in \psi(L)\left(\mathbb{T}^{d}\right), \int_{\mathscr{E} d}\left|\mathbf{v}_{j}(x)\right| d x=1$, $\left\|\mathbf{K}_{j}\right\|_{2}>2 \int_{\mathscr{E} d} \psi\left(\left|\mathbf{v}_{j}(x)\right|\right) d x \geqslant 2 \quad \forall j \in \mathbb{N}$.

Тогда если последовательность $\left\{\mathbf{K}_{j} * g\right\}_{j=1}^{\infty}$ сходится по мере для каждой $g \in \psi(L)\left(\mathbb{T}^{d}\right)$, то найдется постоянная $C>0$ такая, что для всех $j \in \mathbb{N}$ выполняется неравенство

$$
\left\|\mathbf{K}_{j} * \mathbf{v}_{j}\right\|_{1} \leqslant C \int_{\mathscr{E} d} \psi\left(\left|\mathbf{v}_{j}(x)\right|\right) d x \ln \left(\left\|\mathbf{K}_{j}\right\|_{2}\left(\int_{\mathscr{E}^{d}} \psi\left(\left|\mathbf{v}_{j}(x)\right|\right) d x\right)^{-1}\right) .
$$

4.4. Перейдем к формулировке и доказательству основных результатов параграф̆а.

Tеорема 4.1. Пусть вектор $\alpha \in \mathbb{R}^{d}$ строго $\beta$-диофантов, $\beta>0, \theta \in \Theta$. Тогда найдется последовательность $\left\{n_{k}\right\}_{k=1}^{\infty} \subset \mathbb{N}$ такая, что для всякой ее подпоследовательности $\left\{m_{j}\right\}_{j=1}^{\infty} \subset\left\{n_{k}\right\}_{k=1}^{\infty}$ существует функиия $f \in I_{d+\beta}^{\theta} L\left(\mathbb{T}^{d}\right), \quad \int_{\mathscr{E} d} f(x) d x=0$, для которой биркгофовь сумми

$$
\mathbf{B}_{m_{j}}^{\alpha}(f)=\sum_{s=0}^{m_{j}-1} f \circ T_{\alpha}^{s}
$$

расходятся по мере.

ДокАЗАТЕЛЬСТво. Докажем, что сушествуют последовательность натуральных чисел $\left\{n_{k}\right\}_{k=1}^{\infty}$, последовательность тригонометрических полиномов $\left\{R_{k}\right\}_{k=1}^{\infty}$, $\left\|R_{k}\right\|_{1}=1$, и положительные постоянные $C_{1}, C_{2}$ такие, что

$$
\begin{gathered}
\left\|K_{n_{k}} * R_{k}\right\|_{2} \leqslant C_{1} \sqrt{k}, \\
\mid K_{n_{k}} * R_{k} \|_{1} \geqslant C_{2} \sqrt{k}
\end{gathered}
$$

при всех $k \in \mathbb{N}$.

Пусть векторы $q_{s}, s=1,2, \ldots$, удовлетворяют неравенству (3). Переходя в случае необходимости к подпоследовательности, полагаем, что $\left|q_{s+1}\right| /\left|q_{s}\right| \geqslant 4$ при всех $s \in \mathbb{N}$.

Зафиксируем натуральное $N$. Так как

$$
\left|\widehat{K}_{N}(q)\right| \leqslant \frac{|\sin \pi N \alpha q|}{|q|^{d+\beta}|\sin \pi \alpha q|} \leqslant \frac{1}{|q|^{d+\beta}\|\alpha q\|}=\lambda_{\alpha, \beta}(q)
$$

то, полагая $\lambda(q)=\widehat{K}_{N}(q)$, из леммы 3.1 заключаем, что существует $C>0$ такое, что $\lambda^{*}\left(s ; \Delta_{j}\right) \leqslant C s^{-1-\beta / d}$ при всех $j \in \mathbb{N}$ и $s=1, \ldots, \operatorname{card} \Delta_{j}$.

Введем обозначения:

$$
\begin{gathered}
\psi(s)=C s^{-1-\beta / d}, \quad \xi(k)=\frac{C}{2^{\beta / d}} 3^{-k / 2} \\
\Xi_{k}=\left\{\left(\delta_{1}, \ldots, \delta_{k}\right): \delta_{j} \in\{-1 ; 0 ; 1\}, j=1, \ldots, k, \sum_{j=1}^{k}\left|\delta_{j}\right|>1\right\} .
\end{gathered}
$$


По лемме 3.5 для каждого $k \in \mathbb{N}, k \geqslant 4$, найдется набор векторов $\left\{q_{1}^{k}, \ldots, q_{k}^{k}\right\} \subset$ $\left\{q_{s}\right\}_{s=1}^{\infty}$ такой, что

$$
\left|\lambda\left(\delta_{1} q_{1}^{k}+\cdots+\delta_{k} q_{k}^{k}\right)\right| \leqslant \frac{C}{2^{\beta / d}} 3^{-k / 2}
$$

при всех $\left(\delta_{1}, \ldots, \delta_{k}\right) \in \Xi_{k}$.

Положим $R_{k}(x)=\prod_{s=1}^{k}\left(1+\cos \pi q_{s}^{k} x\right)$.

Отметим, что:

a) $\widehat{R_{k}}(q) \neq 0$ тогда и только тогда, когда $q$ представимо в виде $q=\sum_{j=1}^{k} \delta_{j} q_{j}^{k}$, $\delta_{j} \in\{-1 ; 0 ; 1\}$

b) $\widehat{R_{k}}\left( \pm q_{s}^{k}\right)=1 / 2, s=1, \ldots, k$;

c) $0 \leqslant \widehat{R_{k}}(q) \leqslant 1$ для любого $q \in \mathbb{Z}^{d}$.

Из леммы 3.4 заключаем, что сушествует такое $n_{k} \in \mathbb{N},\left|q_{k}^{k}\right|^{\beta+d} / C_{\alpha} \leqslant n_{k} \leqslant$ $2\left|q_{k}^{k}\right|^{\beta+d} / C_{\alpha}$, что

$$
\sum_{s=1}^{k} \frac{\sin ^{2} \pi n_{k} q_{s}^{k} \alpha}{\left|q_{s}^{k}\right|^{2(d+\beta)} \sin ^{2} \pi q_{s}^{k} \alpha} \geqslant \frac{1}{4 \pi^{2}\left(C^{\alpha}\right)^{2}} k
$$

Положим

$$
\begin{aligned}
H_{k}^{1}(x) & =\frac{1}{2} \sum_{q= \pm q_{s}^{k}, s=1, \ldots, k} \widehat{K}_{n_{k}}(q) e_{q}(x), \\
H_{k}^{2}(x) & =\sum_{q=\sum_{s=1}^{k} \delta_{s} q_{s}^{k}, \delta \in \Xi_{k}} \widehat{K}_{n_{k}}(q) \widehat{R_{k}}(q) e_{q}(x) .
\end{aligned}
$$

Имеем $K_{n_{k}} * R_{k}(x)=H_{k}^{1}(x)+H_{k}^{2}(x)$ в силу свойств а) и b).

Имея в виду (29) и $\beta$-диофантовость вектора $\alpha$, получаем

$$
\left\|H_{k}^{1}\right\|_{2}^{2}=\frac{1}{4} \sum_{s=1, \ldots, k} 2\left|\widehat{K}_{n_{k}}\left(q_{s}^{k}\right)\right|^{2} \leqslant \frac{1}{2} \sum_{s=1, \ldots, k} \lambda_{\alpha, \beta}^{2}\left(q_{s}^{k}\right) \leqslant \frac{1}{2} \sum_{s=1, \ldots, k} \frac{1}{C_{\alpha}^{2}}=\frac{1}{2 C_{\alpha}^{2}} k .
$$

Из (30) и свойства с) получим, что

$$
\left\|H_{k}^{2}\right\|_{2}^{2} \leqslant \sum_{\delta \in \Xi_{k}}\left(\frac{C}{2^{\beta / d}} 3^{-k / 2}\right)^{2}=\frac{C^{2}}{2^{2 \beta / d}} 3^{-k} \operatorname{card} \Xi_{k} \leqslant \frac{C^{2}}{2^{2 \beta / d}} .
$$

Следовательно,

$$
\left\|K_{n_{k}} * R_{k}\right\|_{2} \leqslant\left\|H_{k}^{1}\right\|_{2}+\left\|H_{k}^{2}\right\|_{2} \leqslant \frac{1}{\sqrt{2} C_{\alpha}} \sqrt{k}+\frac{C}{2^{\beta / d}} \leqslant C_{1} \sqrt{k},
$$

где $C_{1}=1 /\left(\sqrt{2} C_{\alpha}\right)+C /\left(2^{\beta / d}\right)$.

Теперь оценим снизу $L^{1}$-норму полинома $K_{n_{k}} * R_{k}(x)$. Имеем неравенство $\left\|K_{n_{k}} * R_{k}(x)\right\|_{1} \geqslant\left\|H_{k}^{1}\right\|_{1}-\left\|H_{k}^{2}\right\|_{1}$, при этом $\left\|H_{k}^{2}\right\|_{1} \leqslant\left\|H_{k}^{2}\right\|_{2} \leqslant C^{2} / 2^{2 \beta / d}$.

Для оценки снизу $\left\|H_{k}^{1}\right\|_{1}$ используем свойство логарифмической выпуклости норм в пространствах $L_{p}$, из которого, в частности, следует, что

$$
\left\|H_{k}^{1}\right\|_{2} \leqslant\left\|H_{k}^{1}\right\|_{1}^{1 / 3}\left\|H_{k}^{1}\right\|_{4}^{2 / 3}
$$


а значит, $\left\|H_{k}^{1}\right\|_{1} \geqslant\left\|H_{k}^{1}\right\|_{2}^{3}\left\|H_{k}^{1}\right\|_{4}^{-2}$. Из (31) и (25) имеем

$$
\begin{aligned}
\left\|H_{k}^{1}\right\|_{2} & =\frac{1}{2} \sqrt{\sum_{s=1, \ldots, k} 2\left|\widehat{K}_{n_{k}}\left(q_{s}^{k}\right)\right|^{2}} \\
& =\frac{1}{\sqrt{2}} \sqrt{\sum_{s=1}^{k} \frac{\sin ^{2} \pi n_{k} q_{s}^{k} \alpha}{\left|q_{s}^{k}\right|^{2(d+\beta)} \sin ^{2} \pi q_{s}^{k} \alpha}} \geqslant \frac{1}{2 \sqrt{2} \pi C^{\alpha}} \sqrt{k} .
\end{aligned}
$$

Множества $D^{1}=\left\{q_{1}^{k}, \ldots, q_{k}^{k}\right\}$ и $D^{2}=\left\{-q_{1}^{k}, \ldots,-q_{k}^{k}\right\}$ лакунарны в смысле, указанном в формулировке леммы 3.2. Следовательно, из леммы 3.2 получаем, что

$$
\begin{aligned}
\left\|H_{k}^{1}\right\|_{4} & =\frac{1}{2}\left\|\sum_{q \in D^{1}} \widehat{K}_{n_{k}}(q) e_{q}(\cdot)+\sum_{q \in D^{2}} \widehat{K}_{n_{k}}(q) e_{q}(\cdot)\right\|_{4} \\
& \leqslant \frac{1}{2}\left(\left\|\sum_{q \in D^{1}} \widehat{K}_{n_{k}}(q) e_{q}(\cdot)\right\|_{4}+\left\|\sum_{q \in D^{2}} \widehat{K}_{n_{k}}(q) e_{q}(\cdot)\right\|_{4}\right) \\
& \leqslant \frac{1}{2} c^{3} \sqrt{4}\left(\sqrt{\sum_{q \in D^{1}}\left|\widehat{K}_{n_{k}}(q)\right|^{2}}+\sqrt{\sum_{q \in D^{2}}\left|\widehat{K}_{n_{k}}(q)\right|^{2}}\right) \\
& \leqslant \frac{1}{2} c^{3} 2 \sqrt{2} \sqrt{\sum_{q \in D^{1} \cup D^{2}}\left|\widehat{K}_{n_{k}}(q)\right|^{2}}=2 \sqrt{2} c^{3}\left\|H_{k}^{1}\right\|_{2} .
\end{aligned}
$$

Следовательно, из (32) заключаем, что $\left\|H_{k}^{1}\right\|_{4} \leqslant 2 c^{3} \sqrt{k} / C_{\alpha}$. Итак,

$$
\left\|H_{k}^{1}\right\|_{1} \geqslant\left(\frac{1}{2 \sqrt{2} \pi C^{\alpha}} \sqrt{k}\right)^{3}\left(\frac{2 c^{3}}{C_{\alpha}} \sqrt{k}\right)^{-2}=C_{2} \sqrt{k}
$$

где $C_{2}=C_{\alpha}^{2} /\left(2^{13 / 2} \pi^{3}\left(C^{\alpha}\right)^{3}\left(c^{3}\right)^{2}\right)$.

Пусть $\left\{m_{j}\right\}_{j=1}^{\infty} \subset\left\{n_{k}\right\}_{k=1}^{\infty}$. Предположим, что для любой функции $g \in L\left(\mathbb{T}^{d}\right)$, $\widehat{g}_{0}=0$, последовательность $\left\{K_{m_{j}} * g\right\}_{j=1}^{\infty}$ сходится по мере. В силу того что $\widehat{K}_{m_{j}}(0)=0$, последовательность $\left\{K_{m_{j}} * g\right\}_{j=1}^{\infty}$ сходится по мере для всякой $g \in$ $L\left(\mathbb{T}^{d}\right)$. В силу неравенства Юнга для свертки и неравенства (5) имеем $\left\|K_{m_{j}} * g\right\|_{2} \leqslant$ $\left\|K_{m_{j}}\right\|_{2}\|g\|_{1} \leqslant C_{3} \sqrt{\ln m_{j}}$. Следовательно, $T_{j} g:=K_{m_{j}} * g$ - линейные непрерывные операторы из $L^{1}\left(\mathbb{T}^{d}\right)$ в $L^{2}\left(\mathbb{T}^{d}\right)$ (перестановочные со сдвигами аргумента). Поэтому из теоремы Пичугова заключаем, что существует константа $C$ такая, что

$$
\sup _{0<t<1}\left(t\left(K_{m_{j}} * g\right)^{*}(t)\right) \leqslant C\|g\|_{1}
$$

для любых $j \in \mathbb{N}$ и $g \in L^{1}\left(\mathbb{T}^{d}\right)$.

Определим $k_{j}$ из равенства $n_{k_{j}}=m_{j}$. Полагая в неравенстве $(33) g(x):=R_{k_{j}} *$ $h(x)$, где $h(\cdot)$ - произвольная функция из $L^{1}\left(\mathbb{T}^{d}\right)$, получаем, что

$$
\sup _{0<t<1}\left(t\left(K_{m_{j}} * R_{k_{j}} * h\right)^{*}(t)\right) \leqslant C\left\|R_{k_{j}} * h\right\|_{1} \leqslant C\left\|R_{k_{j}}\right\|_{1}\|h\|_{1}=C\|h\|_{1} .
$$


Отсюда по лемме Пичугова найдутся положительные постоянные $A_{1}$ и $A_{2}$ такие, что для всех $j \in \mathbb{N}$ справедливо неравенство

$$
\left\|K_{m_{j}} * R_{k_{j}}\right\|_{1} \leqslant A_{1}+A_{2} \ln \left(e+\left\|K_{m_{j}} * R_{k_{j}}\right\|_{2}\right) .
$$

Однако последнее утверждение противоречит совместному выполнению неравенств $(27),(28)$.

Полученное противоречие доказывает, что существует функция $\widetilde{g} \in L^{1}\left(\mathbb{T}^{d}\right)$, $\widehat{\widetilde{g}}(0)=0$, для которой последовательность $\left\{K_{m_{j}} * \widetilde{g}\right\}_{j=1}^{\infty}$ расходится по мере. Так как по определению $K_{m_{j}} * \widetilde{g}(x)=\sum_{s=0}^{m_{j}-1} I_{d+\beta}^{\theta} \widetilde{g}\left(T_{\alpha}^{s} x\right)$, то функция $f(\cdot):=$ $I_{d+\beta}^{\theta} \widetilde{g}(\cdot)$ искомая.

Теорема 4.2. Пусть вектор $\alpha \in \mathbb{R}^{d}$ плохо приближаемый, $\theta \in \Theta$. Тогда найдется последовательность $\left\{n_{k}\right\}_{k=1}^{\infty} \subset \mathbb{N}$ такая, что для всякой ее подпоследовательности $\left\{m_{j}\right\}_{j=1}^{\infty} \subset\left\{n_{k}\right\}_{k=1}^{\infty}$ и всякой $\psi \in \Psi$, удовлетворяющей условию $\psi(y)=o\left(y \ln ^{1 / 2} y\right), y \rightarrow+\infty$, существует функиия $f \in I_{d}^{\theta} \psi(L)\left(\mathbb{T}^{d}\right)$, $\int_{\mathscr{E}_{d}} f(x) d x=0$, для которой биркгофовь сумми $\mathbf{B}_{m_{j}}^{\alpha}(f)$ расходятся по мере.

ДокАЗАТЕЛЬСТво. Пусть $\mathscr{F}_{n}(x)-d$-мерное ядро Фейера порядка $n, \mathscr{F}_{n}(x)=$ $\prod_{j=1}^{d} \mathscr{F}_{n}^{j}\left(x_{j}\right), \mathscr{F}_{n}^{j}\left(x_{j}\right)=1+2 \sum_{k=1}^{n}(1-k / n) \cos 2 \pi k x_{j}$. Из известных свойств одномерного ядра Фейера $\mathscr{F}_{n}^{j}$ немедленно следует, что $\mathscr{F}_{n}(x) \geqslant 0, \int_{\mathscr{E} d} \mathscr{F}_{n}(x) d x=1$, $\max _{x} \mathscr{F}_{n}(x)=\mathscr{F}_{n}(0)=n^{d}$.

Введем обозначение $\widetilde{\psi}(y)=\psi(y) / y$. Из выпуклости монотонной функции $\psi(y)$ и условия $\psi(0)=0$ следует монотонное неубывание на $(0,+\infty)$ функции $\widetilde{\psi}(y)$, в силу которого $\widetilde{\psi}\left(\mathscr{F}_{n}(x)\right) \leqslant \widetilde{\psi}\left(n^{d}\right)$ и, значит,

$$
\int_{\mathscr{E} d} \psi\left(\left|\mathscr{F}_{n}(x)\right|\right) d x=\int_{\mathscr{E} d} \mathscr{F}_{n}(x) \widetilde{\psi}\left(\mathscr{F}_{n}(x)\right) d x \leqslant \widetilde{\psi}\left(n^{d}\right) .
$$

В силу леммы Стечкина [15] справедливо неравенство $\mathscr{F}_{n}^{j}\left(x_{j}\right) \geqslant n \cos 2 \pi n x_{j} \geqslant$ $n\left(1-4 n x_{j}\right)$ при $\left|x_{j}\right| \leqslant 1 /(4 n)$. Поэтому

$$
\begin{aligned}
\int_{\mathscr{E} d} \psi\left(\mathscr{F}_{n}(x)\right) d x & \geqslant \int_{0}^{1 /(4 n)} \cdots \int_{0}^{1 /(4 n)} \psi\left(n^{d} \prod_{j=1}^{d}\left(1-4 n x_{j}\right)\right) d x_{1} \cdots d x_{d} \\
& =\frac{1}{(4 n)^{d}} \frac{1}{n^{d}} \int_{0}^{n} \cdots \int_{0}^{n} \psi\left(\prod_{j=1}^{d} x_{j}\right) d x_{1} \cdots d x_{d}
\end{aligned}
$$

Докажем индукцией по $d$ неравенство

$$
\frac{1}{n^{d}} \int_{0}^{n} \cdots \int_{0}^{n} \psi\left(\prod_{j=1}^{d} x_{j}\right) d x_{1} \cdots d x_{d} \geqslant \psi\left(\frac{n^{d}}{2^{d}}\right) .
$$

При $d=1$ применим к функции $\psi$ интегральное неравенство Йенсена:

$$
\frac{1}{n} \int_{0}^{n} \psi(u) d u \geqslant \psi\left(\frac{1}{n} \int_{0}^{n} u d u\right)=\psi\left(\frac{n}{2}\right) .
$$


Пусть $a \geqslant 0$. Введем обозначение $\psi_{a}(y)=\psi(a y)$. Ясно, что функция $\psi_{a}$ выпуклая на $[0,+\infty)$. Предположим, что неравенство (36) справедливо для $d=1, \ldots, d_{0}$. Докажем его справедливость для $d=d_{0}+1$. Имеем

$$
\begin{aligned}
& \frac{1}{n^{d_{0}+1}} \int_{0}^{n} \cdots \int_{0}^{n} \psi\left(\prod_{j=1}^{d_{0}+1} x_{j}\right) d x_{1} \cdots d x_{d_{0}+1} \\
& \quad=\frac{1}{n} \int_{0}^{n} d x_{1} \frac{1}{n^{d_{0}}} \int_{0}^{n} \cdots \int_{0}^{n} \psi_{x_{1}}\left(\prod_{j=1}^{d_{0}} x_{j}\right) d x_{1} \cdots d x_{d_{0}} \\
& \geqslant \frac{1}{n} \int_{0}^{n} \psi_{x_{1}}\left(\frac{n^{d_{0}}}{2^{d_{0}}}\right) d x_{1}=\frac{1}{n} \int_{0}^{n} \psi_{n^{d_{0}} / 2^{d_{0}}}\left(x_{1}\right) d x_{1} \\
& \geqslant \psi_{n^{d_{0} / 2^{d_{0}}}}\left(\frac{n}{2}\right)=\psi\left(\frac{n^{d_{0}+1}}{2^{d_{0}+1}}\right) .
\end{aligned}
$$

Из вогнутости монотонной функции $\psi(\sqrt{y})$ и условия $\psi(0)=0$ следует монотонное невозрастание функции $\psi(\sqrt{y}) / y$ на $(0,+\infty)$. Отсюда $\psi(y) / y^{2} \geqslant \psi\left(2^{d} y\right) /\left(2^{2 d} y^{2}\right)$ и, значит,

$$
\psi\left(n^{d}\right)=\psi\left(2^{d} \frac{n^{d}}{2^{d}}\right) \leqslant 2^{2 d} \psi\left(\frac{n^{d}}{2^{d}}\right) .
$$

Из (35), (36) и последнего неравенства заключаем, что

$$
\int_{\mathscr{E} d} \psi\left(\mathscr{F}_{n}(x)\right) d x \geqslant \frac{1}{(4 n)^{d}} \frac{1}{4^{d}} \psi\left(n^{d}\right)=\frac{1}{4^{2 d}} \widetilde{\psi}\left(n^{d}\right) .
$$

Из теоремы Дирихле о совместном приближении линейных форм [4; гл. 2, §1] следует, что для всякого плохо приближаемого $d$-мерного вектора существуют последовательность векторов $\left\{q_{k}\right\}_{k=1}^{+\infty} \subset \mathbb{Z}^{d},\left|q_{1}\right|<\left|q_{2}\right|<\cdots$, и константы $C_{1}>0$, $E>0$ такие, что $\left|q_{k}\right|^{d}\left\|\alpha q_{k}\right\| \leqslant C_{1}$ и $\left|q_{k}\right| \leqslant E^{k}$ при всех $k \in \mathbb{N}$.

Из леммы 3.4 следует, что для любого $k \in \mathbb{N}$ найдется $n_{k}$ такое, что $C_{2}\left|q_{k}\right|^{d} \leqslant$ $n_{k} \leqslant 2 C_{2}\left|q_{k}\right|^{d}$ и

$$
\sum_{s=1}^{k} \frac{\sin ^{2} \pi n_{k} q_{s} \alpha}{\left|q_{k}\right|^{2 d} \sin ^{2} \pi q_{s} \alpha} \geqslant C_{3} k,
$$

где константы $C_{2} \geqslant 1$ и $C_{3}>0$ не зависят от выбора $k .^{5}$

Пусть $\left\{m_{j}\right\}_{j=1}^{\infty}-$ произвольная подпоследовательность $\left\{n_{k}\right\}_{k=1}^{\infty}$. Определим $k_{j}$ из равенства $m_{j}=n_{k_{j}}$. Введем обозначения: $\mathbf{K}_{j}=K_{m_{j}}, \mathbf{v}_{j}=\mathscr{F}_{m_{j}}$. Через $C_{4}, C_{5}, \ldots, C_{13}$ будем обозначать положительные постоянные, не зависяшие от выбора $j$.

В силу леммы 4.1 сушествует константа $C_{4}>0$ такая, что

$$
\left\|\mathbf{K}_{j}\right\|_{2}=\left\|K_{m_{j}}\right\|_{2} \leqslant C_{4} \sqrt{\ln m_{j}}
$$

\footnotetext{
${ }^{5}$ Из леммы 3.4 имеем $C_{2}=1 / C_{\alpha}$, где $C_{\alpha}$ - из неравенства (2). В силу теоремы Дирихле $C_{\alpha} \leqslant 1$
} 
а из (38) следует, что

$$
\left\|\mathbf{K}_{j}\right\|_{2}^{2}=\left\|K_{m_{j}}\right\|_{2}^{2} \geqslant \sum_{s=1}^{k_{j}}\left|\widehat{K}_{m_{j}}\left(q_{s}\right)\right|^{2}=\sum_{s=1}^{k_{j}} \frac{\sin ^{2} \pi m_{j} q_{s} \alpha}{\left|q_{k}\right|^{2 d} \sin ^{2} \pi q_{s} \alpha} \geqslant C_{3} k_{j} .
$$

Оценим $\left\|\mathbf{K}_{j} * \mathbf{v}_{j}\right\|_{2}$ снизу. Имеем

$$
\left\|\mathbf{K}_{j} * \mathbf{v}_{j}\right\|_{2}^{2} \geqslant \sum_{s=1}^{k_{j}-2}\left|\widehat{K}_{m_{j}}\left(q_{s}\right)\right|^{2}\left|\widehat{\mathscr{F}}_{m_{j}}\left(q_{s}\right)\right|^{2}
$$

Обозначим через $q_{s}^{j}$ компоненты вектора $q_{s}$. Тогда

$$
\left|\widehat{\mathscr{F}}_{m_{j}}(q)\right|^{2}=\prod_{j=1}^{d}\left|1-\frac{q_{s}^{j}}{m_{j}}\right|^{2} \geqslant \prod_{j=1}^{d}\left|1-\frac{\left|q_{s}\right|}{m_{j}}\right|^{2}=\left|1-\frac{\left|q_{s}\right|}{m_{j}}\right|^{2 d}
$$

Ясно, что для $s=1, \ldots, k_{j}-2$ справедливы неравенства

$$
\frac{\left|q_{s}\right|}{m_{j}} \leqslant \frac{\left|q_{k_{j}-2}\right|}{C_{2}\left|q_{k_{j}}\right|^{d}} \leqslant \frac{\left|q_{k_{j}-2}\right|}{\left|q_{k_{j}}\right|^{d}} \leqslant \frac{1}{2} .
$$

Значит,

$$
\left\|\mathbf{K}_{j} * \mathbf{v}_{j}\right\|_{2} \geqslant \frac{1}{2^{d}} \sqrt{\sum_{s=1}^{k_{j}-2}\left|\widehat{K}_{m_{j}}\left(q_{s}\right)\right|^{2}} \geqslant C_{5} \sqrt{k_{j}}
$$

Оценим $\left\|\mathbf{K}_{j} * \mathbf{v}_{j}\right\|_{4}$ сверху. Имеем $\left|\widehat{K}_{m_{j}}(q)\right| \leqslant \lambda_{\alpha, 0}(q), q \in \mathbb{Z}^{d}$. Из леммы 3.1 следует, что $\lambda_{\alpha, 0}^{*}\left(s ; \Delta_{j}\right) \leqslant C / s, j \in \mathbb{Z}_{+}, s=1, \ldots, \operatorname{card} \Delta_{j}$. Значит, полагая $\lambda(k)=\widehat{K}_{m_{j}}(k), g=\mathbf{v}_{j}$, применяя лемму 3.3 и неравенство $(34)$, получаем, что

$$
\begin{aligned}
\left\|\mathbf{K}_{j} * \mathbf{v}_{j}\right\|_{4} & =\left\|\sum_{k \neq 0} \lambda(k) \widehat{g}_{k} e_{k}(\cdot)\right\|_{4} \leqslant C_{6} \int_{\mathscr{E} d}\left|\mathscr{F}_{m_{j}}(x)\right| \ln ^{1 / 2}\left(e+\left|\mathscr{F}_{m_{j}}(x)\right|\right) d x+C_{7} \\
& \leqslant C_{8} \ln ^{1 / 2}\left(m_{j}^{d}\right) .
\end{aligned}
$$

Имеем соотношения: $\ln m_{j}=\ln n_{k_{j}} \leqslant \ln \left(2 C_{2}\left|q_{k_{j}}\right|^{d}\right) \leqslant \ln \left(2 C_{2} E^{d k_{j}}\right) \leqslant C_{9} k_{j}$. Отсюда, применяя (42), получаем оценку

$$
\left\|\mathbf{K}_{j} * \mathbf{v}_{j}\right\|_{4} \leqslant C_{10} \sqrt{k_{j}}
$$

Для оценки снизу $\left\|\mathbf{K}_{j} * \mathbf{v}_{j}\right\|_{1}$ воспользуемся неравенством $\|h\|_{1} \geqslant\|h\|_{2}^{3}\|h\|_{4}^{-2}$, справедливым для любой функции $h \in L^{4}\left(\mathbb{T}^{d}\right),\|h\|_{4} \neq 0$. Из (41) и (43) заключаем, что

$$
\left\|\mathbf{K}_{j} * \mathbf{v}_{j}\right\|_{1} \geqslant C_{11} \sqrt{k_{j}}
$$


Из неравенств (39), (34), (37) и (44) следует, что

$$
\begin{gathered}
\frac{1}{\left\|\mathbf{K}_{j} * \mathbf{v}_{j}\right\|_{1}} \int_{\mathscr{E} d} \psi\left(\left|\mathbf{v}_{j}(x)\right|\right) d x \ln \left(\left\|\mathbf{K}_{j}\right\|_{2}\left(\int_{\mathscr{E} d} \psi\left(\left|\mathbf{v}_{j}(x)\right|\right) d x\right)^{-1}\right) \\
\leqslant C_{12} \frac{\widetilde{\psi}\left(m_{j}^{d}\right)}{\sqrt{k_{j}}} \ln \frac{C_{13} \sqrt{k_{j}}}{\widetilde{\psi}\left(m_{j}^{d}\right)}
\end{gathered}
$$

Из условия теоремы следует, что $\widetilde{\psi}\left(m_{j}^{d}\right)=o\left(\sqrt{k_{j}}\right)$ при $j \rightarrow \infty$, а так как

$$
u \ln \frac{C_{13}}{u}=o(1)
$$

при $u \rightarrow 0+$, то из $(45)$ заключаем, что

$$
\frac{1}{\left\|\mathbf{K}_{j} * \mathbf{v}_{j}\right\|_{1}} \int_{\mathscr{E} d} \psi\left(\left|\mathbf{v}_{j}(x)\right|\right) d x \ln \left(\left\|\mathbf{K}_{j}\right\|_{2}\left(\int_{\mathscr{E} d} \psi\left(\left|\mathbf{v}_{j}(x)\right|\right) d x\right)^{-1}\right) \rightarrow 0
$$

при $j \rightarrow \infty$. Из неравенств $(40),(34),(37)$ и условия на функцию $\psi$ следует, что

$$
\left\|\mathbf{K}_{j}\right\|_{2}>2 \int_{\mathscr{E} d} \psi\left(\left|\mathbf{v}_{j}(x)\right|\right) d x \geqslant 2
$$

для всех $j$, превосходяших некоторое $j_{0} \in \mathbb{N}$.

Применяя лемму 4.2 , из неравенств (46), (47) делаем вывод о сушествовании функции $g_{0} \in \psi(L)\left(\mathbb{T}^{d}\right)$, для которой последовательность $\left\{\mathbf{K}_{j} * g_{0}\right\}_{j=1}^{\infty}$ расходится по мере, а значит, полагая $f=I_{d}^{\theta} * g$, заключаем, что последовательность $\left\{\sum_{s=0}^{m_{j}-1} f\left(T_{\alpha}^{s} x\right)\right\}_{j=1}^{\infty}$ расходится по мере, и при этом

$$
f \in I_{d}^{\theta} \psi(L)\left(\mathbb{T}^{d}\right), \quad \int_{\mathscr{E} d} f(x) d x=0
$$

Лемма 4.3. Пусть $\alpha \in \mathbb{R}^{d}$ - существенно иррачиональньй вектор, $\left\{m_{j}\right\}_{j=1}^{\infty}$ - $\alpha$-допустимая последовательность, $H$ - подпространство $L^{0}\left(\mathbb{T}^{d}\right)_{0}$, содержащее в себе $\mathscr{T}_{0}^{d}$. Предположим, что существует функиия $f_{0} \in H$ такая, что последовательность биркгофовых сумм $\mathbf{B}_{m_{j}}^{\alpha}\left(f_{0}\right), j=1,2, \ldots$, расходится по мере. Тогда существует непустое множество $V \subset H$, удовлетворяющее следующим условиям:

1) для каждой функции $f \in V$ суммы $\mathbf{B}_{m_{j}}^{\alpha}(f), j=1,2, \ldots$, расходятся по мере;

2) $V$ есть $G_{\delta}$-подмножество $\left(H, \rho_{0}\right)$;

3) если $f \in V$, mo $\left\{f+p: p \in \mathscr{T}_{0}^{d}\right\} \subset V$. 
ДокаЗАТЕЛЬСТво. Положим $P(f, \lambda)=\operatorname{mes}\{x:|f(x)|>\lambda\}$. Обозначим $B^{j}(f)=\mathbf{B}_{m_{j}}^{\alpha}(f)$, а через $U$ обозначим совокупность функций $f \in H$, для которых $B^{j}(f)$ расходится по мере. В силу критерия Коши сходимости последовательности множество $U$ представимо в виде $U=\bigcup_{k \in \mathbb{N}, s \in \mathbb{N}} U^{k, s}$, где $U^{k, s}=\bigcap_{N \in \mathbb{N}} U_{N}^{k, s}$,

$$
U_{N}^{k, s}=\left\{f \in H: \exists n, l \geqslant N, P\left(B^{n}(f)-B^{l}(f), \frac{1}{k}\right)>\frac{1}{s}\right\} .
$$

Так как $U \neq \varnothing$, то $U^{k_{0}, s_{0}} \neq \varnothing$ хотя бы для одной пары $k_{0}, s_{0}$.

Пусть $f_{0} \in U^{k_{0}, s_{0}}$. Для каждого $N \in \mathbb{N}$ определим $n(N), l(N) \geqslant N$ из условия $P\left(B^{n(N)}\left(f_{0}\right)-B^{l(N)}\left(f_{0}\right), 1 / k_{0}\right)>1 / s_{0}$.

Пусть $p$ - действительнозначный тригонометрический полином с нулевьм средним, и пусть полином $\tau$ удовлетворяет уравнению $p(x)=\tau(x+\alpha)-\tau(x)$. Имеем:

$$
B^{n}\left(f_{0}+p\right)(x)-B^{l}\left(f_{0}+p\right)(x)=B^{n}\left(f_{0}\right)(x)-B^{l}\left(f_{0}\right)(x)+\tau\left(T_{\alpha}^{m_{n}} x\right)-\tau\left(T_{\alpha}^{m_{l}} x\right) .
$$

Из условия $\alpha$-допустимости последовательности $\left\{m_{j}\right\}_{j=1}^{\infty}$ следует, что $R_{n, l}(p):=$ $\left\|\tau \circ T_{\alpha}^{m_{n}}-\tau \circ T_{\alpha}^{m_{l}}\right\|_{\infty} \rightarrow 0$ при $j \rightarrow \infty$ и, значит, сушествует $N=N(p)$ такое, что $R_{n, l}(p)<1 /\left(2 k_{0}\right)$ при всех $n, l \geqslant N$. Отсюда и из условия на функцию $f$ следует, что

$$
\begin{aligned}
P\left(B^{n(N)}\left(f_{0}+p\right)-B^{l(N)}\left(f_{0}+p\right), \frac{1}{2 k_{0}}\right) & \\
& \geqslant P\left(B^{n(N)}\left(f_{0}\right)-B^{l(N)}\left(f_{0}\right), \frac{1}{2 k_{0}}+R_{n(N), l(N)}(p)\right) \\
& \geqslant P\left(B^{n(N)}\left(f_{0}\right)-B^{l(N)}\left(f_{0}\right), \frac{1}{k_{0}}\right)>\frac{1}{s_{0}}
\end{aligned}
$$

при всех $N \geqslant N_{0}$. Следовательно, $f_{0}+p \in U^{2 k_{0}, s_{0}}$.

Докажем теперь, что каждое из множеств $U_{N}^{2 k_{0}, s_{0}}, N \in \mathbb{N}$, открыто в $\left(H, \rho_{0}\right)$.

Пусть $f \in U_{N}^{2 k_{0}, s_{0}}$, натуральные $n=n(N)$ и $l=l(N)$ определены из условий $P\left(B^{n}(f)-B^{l}(f), 1 /\left(2 k_{0}\right)\right)=: E_{f}>1 / s_{0}, n>l \geqslant N$. Положим $\sigma_{0}=E_{f}-1 / s_{0}$.

Имеем

$$
\begin{aligned}
& P\left(B^{n}(f+g)-B^{l}(f+g), \frac{1}{2 k_{0}}\right) \\
& \quad \geqslant \operatorname{mes}\left\{x:\left|B^{n}(f)(x)-B^{l}(f)(x)\right|>\frac{1}{2 k_{0}}+\left|B^{n}(g)(x)-B^{l}(g)(x)\right|\right\} .
\end{aligned}
$$

Пусть $\delta_{0}>0$ выбрана так, чтобы $P\left(B^{n}(f)-B^{l}(f), 1 /\left(2 k_{0}\right)+\delta_{0}\right)>1 / s_{0}+\sigma_{0} / 2$.

Имеем

$$
\begin{gathered}
\operatorname{mes}\left\{x:\left|B^{n}(f)(x)-B^{l}(f)(x)\right|>\frac{1}{2 k_{0}}+\left|B^{n}(g)(x)-B^{l}(g)(x)\right|\right\} \\
\geqslant P\left(B^{n}(f)-B^{l}(f), \frac{1}{2 k_{0}}+\delta_{0}\right)-P\left(B^{n}(g)-B^{l}(g), \delta_{0}\right) .
\end{gathered}
$$


Так как

$$
\begin{aligned}
P\left(B^{n}(g)-B^{l}(g), \delta_{0}\right) & \leqslant \operatorname{mes}\left\{x: \sum_{s=l}^{n-1}\left|g\left(T_{\alpha}^{s} x\right)\right|>\delta_{0}\right\} \leqslant \sum_{s=l}^{n-1} P\left(g \circ T_{\alpha}^{s}, \frac{\delta_{0}}{n-l}\right) \\
& =(n-l) P\left(g, \frac{\delta_{0}}{n-l}\right)
\end{aligned}
$$

и $P(g, \lambda) \leqslant(1+1 / \lambda) \rho_{0}(g, 0)$ для всякого $\lambda>0$, то $P\left(B^{n}(g)-B^{l}(g), \delta_{0}\right)<\sigma_{0} / 2$ всякий раз, когда $\rho_{0}(g, 0)<\left(1+(n-l) / \delta_{0}\right)^{-1}\left(\sigma_{0} /(2(n-l))\right)$, а следовательно, $P\left(B^{n}(f+g)-B^{l}(f+g), 1 /\left(2 k_{0}\right)\right)>1 / s_{0}$ для таких $g \in H$.

Tеорема 4.3. Пусть вектор $\alpha \in \mathbb{R}^{d}$ строго $\beta$-диофантов, $\theta \in \Theta, H=$ $I_{d+\beta}^{\theta} L\left(\mathbb{T}^{d}\right)_{0}$, если $\beta>0, \quad H=I_{d}^{\theta} \psi(L)\left(\mathbb{T}^{d}\right)_{0}, \psi \in \Psi, \psi(y)=o\left(y \ln ^{1 / 2} y\right), y \rightarrow \infty$, если $\beta=0$;

Тогда существует $\alpha$-допустимая последовательность $\left\{m_{j}\right\}_{j=1}^{\infty}$ такая, что для типичной функции $f$ из пространства $\left(H, \rho_{0}\right)$ биркгофовьь сум⿻ми $\mathbf{B}_{m_{j}}^{\alpha}(f)$, $j=1,2, \ldots$, расходятся по мере.

ДокАЗАТЕЛЬСТво. Пусть последовательность $\left\{n_{k}\right\}_{k=1}^{\infty} \subset \mathbb{N}$ удовлетворяет теореме 4.1 в случае $\beta>0$ и теореме 4.2 , если $\beta=0$.

Выберем из нее $\alpha$-допустимую подпоследовательность $\left\{m_{j}\right\}_{j=1}^{\infty}$.

По теоремам 4.1 и 4.2 сушествует функция $f_{0} \in H$, для которой биркгофовы суммы $\mathbf{B}_{m_{j}}^{\alpha}\left(f_{0}\right)$ расходятся по мере.

Для завершения доказательства теоремы осталось воспользоваться леммой 4.3 .

\section{$\S 5$. О разрешимости аддитивного когомологического уравнения для сдвигов тора}

Нетрудно видеть, что любой полином $p \in \mathscr{T}^{d}$ является тривиальным аддитивньм коциклом над $T_{\alpha}$ для всякого сушественно иррационального вектора $\alpha$. С другой стороны, для суммируемой на $\mathbb{T}^{d}$ функции, отличной от тригонометрического полинома, разрешимость уравнения (1) зависит от ее "гладкости" и от диофантовых свойств вектора $\alpha$.

TеОРема 5.1. Пусть вектор $\alpha \in \mathbb{R}^{d} \quad \beta$-диофантов, $X=I_{d+\beta} L \log _{+}^{1 / 2} L\left(\mathbb{T}^{d}\right)$, $d \in \mathbb{N}$, или $X=W^{1+\beta} L \log _{+}^{1 / 2} L(\mathbb{T})$, если $d=1$.

Тогда всякая функиия $f$ из пространства $X$ есть тривиальньй аддитивньй коцикл над $T_{\alpha}$.

При этом для решения $w$ уравнения (1) верно включение $w \in \exp \left(L^{2}\right)\left(\mathbb{T}^{d}\right)$, если $\beta>0$, и включение $w \in \exp \left(L^{2 / 3}\right)\left(\mathbb{T}^{d}\right)$, если $\beta=0$.

ДокаЗАтЕльство. Определим функцию $\lambda: \mathbb{Z}^{d} \rightarrow \mathbb{C}$ по формуле $\lambda(k)=\theta(k) \times$ $\left(1+|k|_{2}^{2}\right)^{-(d+\beta) / 2}\left(e_{k}(\alpha)-1\right)^{-1}$, где $\theta(0)=0$, а при $k \neq 0$ полагаем $\theta(k) \equiv 1$, если $X=I_{d+\beta} L \log _{+}^{1 / 2} L\left(\mathbb{T}^{d}\right)$, и $\theta(k)=\exp \left(-\frac{\pi}{2} i r \operatorname{sign} k\right)$, если $X=W^{1+\beta} L \log _{+}^{1 / 2} L(\mathbb{T})$. Ясно, что

$$
|\lambda(k)| \leqslant \frac{1}{2}|k|^{-d-\beta}|\sin \pi \alpha k|^{-1} \leqslant \frac{1}{4}|k|^{-d-\beta}\|\alpha k\|^{-1}=\frac{1}{4} \lambda_{\alpha, \beta}(k) .
$$


Отсюда и из леммы 3.1 следует, что сушествует положительная константа $c$ такая, что $\lambda^{*}\left(s ; \Delta_{j}\right) \leqslant c / s^{1+\beta / d}$ при $s=1, \ldots, \operatorname{card} \Delta_{j}, j \in \mathbb{N}$.

Пусть $g \in L \log _{+}^{1 / 2} L\left(\mathbb{T}^{d}\right), \widehat{g}_{0}=0$. В силу леммы 3.3 величины $\lambda(k) \widehat{g}_{k}, k \in \mathbb{Z}^{d}$, являются коэффициентами Фурье функции $\Lambda g \in \bigcap_{2 \leqslant q<\infty} L^{q}\left(\mathbb{T}^{d}\right)$.

Пусть последовательность $g_{n} \in L \log _{+}^{1 / 2} L\left(\mathbb{T}^{d}\right), n=1,2, \ldots$, сходится в пространстве $L \log _{+}^{1 / 2} L\left(\mathbb{T}^{d}\right)$ к некоторой функции $\widetilde{g}$, а последовательность $f_{n}:=\Lambda g_{n}$, $n=1,2, \ldots$, сходится в пространстве $L^{2}\left(\mathbb{T}^{d}\right)$ к функции $\widetilde{f}$. Ясно, что тогда для каждого $k \in \mathbb{Z}^{d}$ справедливо $\widehat{g}_{n}(k) \rightarrow \widehat{\widetilde{g}}(k)$ и $\widehat{f}_{n}(k) \rightarrow \widehat{\widetilde{f}}(k), n \rightarrow \infty$. Но согласно определению $\Lambda g_{n}$ мы имеем равенство $\widehat{f}_{n}(k)=\lambda(k) \widehat{g}_{n}(k)$ при каждом $k$. Отсюда, совершая предельньй переход при $n \rightarrow \infty$, получаем, что $\widehat{\widetilde{f}}(k)=\lambda(k) \widehat{\widetilde{g}}(k)$ и, значит, $\widetilde{f}(\cdot)=\Lambda \widetilde{g}(\cdot)$. Следовательно, по теореме Банаха о замкнутом графике $\Lambda$ линейный непрерывный оператор из $L \log _{+}^{1 / 2} L\left(\mathbb{T}^{d}\right)$ в $L^{2}\left(\mathbb{T}^{d}\right)$.

Из определения $\Lambda$ немедленно следует, что если $\tau(\cdot) \in \mathscr{T}^{d}, \widehat{\tau}_{0}=0$, то

$$
\begin{aligned}
& (\Lambda \tau)(x+\alpha)-\Lambda \tau(x)=\sum_{k} \lambda(k) \widehat{\tau}_{k}\left(e_{k}(x+\alpha)-e_{k}(x)\right) \\
& \quad=\sum_{k \neq 0} \lambda(k) \widehat{\tau}_{k}\left(e_{k}(\alpha)-1\right) e_{k}(x)=\sum_{k \neq 0} \widehat{\tau}_{k} \theta(k) \frac{e_{k}(x)}{\left(1+|k|_{2}^{2}\right)^{(d+\beta) / 2}}=I_{d+\beta}^{\theta} * \tau(x) .
\end{aligned}
$$

Пусть опять $g$ - произвольная функция из $L \log _{+}^{1 / 2} L\left(\mathbb{T}^{d}\right)$, удовлетворяюшая условию $\widehat{g}_{0}=0$, и пусть $t_{n}$ - последовательность тригонометрических полиномов, $\widehat{t}_{n}(0)=0$, сходящаяся к $g$ в $L \log _{+}^{1 / 2} L\left(\mathbb{T}^{d}\right)$. Тогда в силу $(48)$ имеем

$$
\begin{aligned}
& \left\|I_{d+\beta}^{\theta} * g(\cdot)-\Lambda g(\cdot+\alpha)+\Lambda g(\cdot)\right\|_{2} \\
& \quad=\left\|I_{d+\beta}^{\theta} *\left(g-t_{n}\right)(\cdot)-\Lambda\left(g-t_{n}\right)(\cdot+\alpha)+\Lambda\left(g-t_{n}\right)(\cdot)\right\|_{2} \\
& \quad \leqslant\left\|I_{d+\beta}^{\theta} *\left(g-t_{n}\right)\right\|_{2}+2\left\|\Lambda\left(g-t_{n}\right)\right\|_{2} \\
& \quad \leqslant\left\|I_{d+\beta}^{\theta}\right\|_{2}\left\|g-t_{n}\right\|_{1}+2\left\|\Lambda_{L \log _{+}^{1 / 2} L \rightarrow L^{2}}\right\| \cdot\left\|g-t_{n}\right\|_{L \log _{+}^{1 / 2} L} \rightarrow 0
\end{aligned}
$$

при $n \rightarrow \infty$. Отсюда заключаем, что $I_{d+\beta}^{\theta} * g(x)=\Lambda g(x+\alpha)-\Lambda g(x)$ при почти всех $x \in \mathbb{T}^{d}$.

Итак, пусть функция $f$ принадлежит пространству $I_{d+\beta}^{\theta} L \log _{+}^{1 / 2} L\left(\mathbb{T}^{d}\right)$. Тогда $f(x)-\int_{\mathscr{E} d} f(t) d t=I_{d+\beta}^{\theta} * g(x)$, где $g \in L \log _{+}^{1 / 2} L\left(\mathbb{T}^{d}\right), \widehat{g}_{0}=0$, и, значит, $f(x)-$ $\int_{\mathscr{E} d} f(t) d t=w(x+\alpha)-w(x)$ при почти всех $x$, где $w=\Lambda g$.

Докажем теперь, что $w \in \exp \left(L^{1 / \varkappa}\right)\left(\mathbb{T}^{d}\right)$, где константа $\varkappa$ - из условия леммы 3.3. Из этой леммы следует, что

$$
\|C w\|_{k / \varkappa}^{k / \varkappa} \leqslant C^{k / \varkappa}\left(\frac{k}{\varkappa}\right)^{k} J^{k / \varkappa}(g)
$$


для любой положительной постоянной $C$ и любого $k \geqslant 2$. Отсюда заключаем, что

$$
\begin{aligned}
\int_{\mathscr{E} d} \exp \left(|C w(x)|^{1 / \varkappa}\right) d x & =1+\int_{\mathscr{E} d}|C w(x)|^{1 / \varkappa} d x+\sum_{k=2}^{\infty} \frac{1}{k !} \int_{\mathscr{E} d}|C w(x)|^{k / \varkappa} d x \\
& \leqslant 1+C\|w\|_{1 / \varkappa}^{1 / \varkappa}+\sum_{k=2}^{\infty} \frac{k^{k}}{k !} R^{k}
\end{aligned}
$$

где $R=(1 / \varkappa) C^{1 / \varkappa} J^{1 / \varkappa}(g)$. Имеем $k^{k} / k ! \leqslant e^{k}$ и $R<e^{-1}$, если $C<(\varkappa / e)^{\varkappa} / J(g)$. Значит, при таком $C$ ряд $\sum_{k=2}^{\infty}\left(k^{k} / k !\right) R^{k}$ сходится, а следовательно, конечен интеграл $\int_{\mathscr{E} d} \exp \left(|C w(x)|^{1 / \varkappa}\right) d x$, что и требовалось.

Заметим, что если $f$ - нетривиальньй аддитивный коцикл над $T_{\alpha}$, то $f+C$, где $C \in \mathbb{R}$ - произвольная постоянная, - также нетривиальный аддитивный коцикл над $T_{\alpha}$. Поэтому в теоремах 5.2 и 5.3 ограничимся рассмотрением функций, имеющих нулевое среднее.

ТеОРема 5.2. Пусть вектор $\alpha \in \mathbb{R}^{d}$ строго $\beta$-диофантов и имеет место любое из следующих четырех условий:

1. $X=I_{d+\beta} L^{1}\left(\mathbb{T}^{d}\right)_{0}, \beta>0, d \in \mathbb{N}$;

2. $X=W^{1+\beta} L^{1}(\mathbb{T})_{0}, \beta>0, d=1$;

3. $X=I_{d} \psi(L)\left(\mathbb{T}^{d}\right)_{0}, \beta=0, d \in \mathbb{N}, \psi \in \Psi, \psi(y)=o\left(y \ln ^{1 / 2} y\right), y \rightarrow+\infty$;

4. $X=W^{1} \psi(L)(\mathbb{T})_{0}, \beta=0, d=1, \psi \in \Psi, \psi(y)=o\left(y \ln ^{1 / 2} y\right), y \rightarrow+\infty$.

Тогда типичная функция из пространства $\left(X, \rho_{0}\right)$ есть нетривиальный аддитивный коцикл над $T_{\alpha}$.

ДокаЗАтЕЛЬСтво. Ниже будем полагать, что $\psi(y)=y$, если $\beta>0$. Определим $\theta$ как в теореме 5.1. По теореме 4.3 сушествует последовательность $\left\{m_{j}\right\}_{j=1}^{\infty}$ такая, что дробные доли $\left\{m_{j} \alpha_{s}\right\}, s=1, \ldots, d$, имеют предел при $j \rightarrow \infty$, а для типичной функции $f \in X$ биркгофовы суммы $\mathbf{B}_{m_{j}}^{\alpha}(f)$ расходятся по мере. Для завершения доказательства осталось только заметить, что совокупность $U$ функций $f \in X$, для которых $\mathbf{B}_{m_{j}}^{\alpha}(f)$ расходится по мере, есть подмножество нетривиальных над $T_{\alpha}$ аддитивных коциклов в пространстве $X$.

TеОРема 5.3. Пусть вектор $\alpha \in \mathbb{R}^{d}$ строго $\beta$-диофантов, $X=I_{d+\beta} C\left(\mathbb{T}^{d}\right)_{0}$, $d$ любое, или $X=W^{1+\beta} C(\mathbb{T})_{0}$, если $d=1$.

Тогда для типичной функции из пространства $\left(X, \rho_{C}\right)$ уравнение (1) не имеет непрерывного решения.

ДокАЗАТЕЛЬСтво. Определим $\theta$ как в теореме 5.1. Пусть последовательность $\left\{m_{j}\right\}_{j=1}^{\infty}$ удовлетворяет условию теоремы 4.3.

Рассуждая “от противного", найдем функцию из $I_{d+\beta}^{\theta} C\left(\mathbb{T}^{d}\right)_{0}$, для которой последовательность биркгофовых сумм $B^{j}(f)=\sum_{s=0}^{m_{j}-1} f \circ T_{\alpha}^{s}$ не является равномерно сходящейся.

Предположим, что для всякой функции $g \in C\left(\mathbb{T}^{d}\right)_{0}$ последовательность $K_{m_{j}} g=$ $B^{j}\left(I_{d+\beta}^{\theta} g\right)$ равномерно сходится и, значит, равномерно ограничена в $C\left(\mathbb{T}^{d}\right)_{0}$. По теореме Банаха-Штейнгауза отсюда заключаем, что сушествует константа $C>0$ такая, что $\left\|K_{m_{j}}\right\|_{1}<C \forall j \in \mathbb{N}$. Пусть $h \in L^{1}\left(\mathbb{T}^{d}\right)_{0}$. Для каждого $\varepsilon>0$ найдется 
функция $\tau \in C\left(\mathbb{T}^{d}\right)_{0}$ такая, что $\|h-\tau\|_{1}<\varepsilon /(4 C)$, а для функции $\tau$ найдется $N \in \mathbb{N}$ такое, что $\left\|K_{m_{l}} * \tau-K_{m_{n}} * \tau\right\|_{C}<\varepsilon / 2$ при всех $n, k>N$. Тогда

$$
\begin{aligned}
\left\|K_{m_{l}} * h-K_{m_{n}} * h\right\|_{1} \leqslant & \left\|K_{m_{l}} *(h-\tau)-K_{m_{n}} *(h-\tau)\right\|_{1} \\
& +\left\|K_{m_{l}} * \tau-K_{m_{n}} * \tau\right\|_{1} \\
\leqslant & 2 C\|h-\tau\|_{1}+\left\|K_{m_{l}} * \tau-K_{m_{n}} * \tau\right\|_{C}<\varepsilon .
\end{aligned}
$$

Отсюда по критерию Коши сходимости последовательности заключаем, что для всякой $h \in L^{1}\left(\mathbb{T}^{d}\right)_{0}$ последовательность $K_{m_{j}} g$ сходится в пространстве $L^{1}\left(\mathbb{T}^{d}\right)_{0}$, а значит, последовательность $B^{j} * f$ сходится по мере для всякой $f \in I_{d+\beta}^{\theta} L^{1}\left(\mathbb{T}^{d}\right)_{0}$, что, однако, противоречит теореме 4.3.

Заметим теперь, что совокупность $U$ функций $f \in I_{d+\beta}^{\theta} C\left(\mathbb{T}^{d}\right)_{0}$, для которых последовательность $B^{j}(f)$ не является равномерно сходящейся, есть подмножество множества функций из $I_{d+\beta}^{\theta} C\left(\mathbb{T}^{d}\right)_{0}$, для которых уравнение $(1)$ не имеет непрерывного решения.

Доказательство того, что $U$ содержит всюду плотное множество типа $G_{\delta}$, проводится по схеме, использованной при доказательстве леммы 4.3 .

Из теоремы 5.3 и теорем вложения $\S 2$ следует, что в следующей известной теореме М. Эрмана [16] число $\varepsilon$ нельзя положить равным нулю, по крайней мере если $d=1$ или $d+\beta \notin \mathbb{N}$.

Теорема (М. Эрман). Пусть $\alpha \in \mathbb{R}^{d}-\beta$-диофантов вектор. Eсли $r=d+$ $\beta+\varepsilon, \varepsilon \in(0,1)$, u $f \in C^{r}\left(\mathbb{T}^{d}\right)$, то уравнение (1) имеет непрерывное решение $w \in C^{\varepsilon}\left(\mathbb{T}^{d}\right)$.

\section{$\S 6$. О замене времени в линейном потоке на торе и изоморфизме специальных потоков}

Напомним, что потоком $R^{t}, t \in \mathbb{R}$, назьвается однопараметрическая группа автоморфизмов пространства Лебега. Поток $R^{t}$ называется измеримым, если каково бы ни было измеримое множество $L$, множество тех точек $(x, t)$ прямого произведения $L \times \mathbb{R}$, для которых $R^{t} x \in L$, измеримо. Пусть на пространстве Лебега $\left(X_{1}, \mu_{1}\right)$ задан измеримый поток $R_{1}^{t}, t \in \mathbb{R}$, а на пространстве Лебега $\left(X_{2}, \mu_{2}\right)$ - измеримый поток $R_{2}^{t}, t \in \mathbb{R}$. Потоки $R_{1}^{t}$ и $R_{2}^{t}$ называются (метрически) изоморфнымии, если сушествуют такие множества полной меры $M_{1} \subset X_{1}$ и $M_{2} \subset X_{2}$ и взаимно однозначное сохраняюшее меру отображение $S$ множества $M_{1}$ на множество $M_{2}$, что $R_{2}^{t}(y)=S \circ R_{1}^{t} \circ S^{-1}(y)$ при всех $t \in \mathbb{R}$ и всех $y \in M_{2}$.

Функция $g \in L^{2}(X)$ называется собственной функцией потока $R^{t}$, заданного на пространстве $X$, если существует действительное $\lambda$ ( собственное значение потока) такое, что $g\left(R^{t} x\right)=e^{2 \pi i \lambda t} g(x)$ для всех $t \in \mathbb{R}$ и почти всех $x \in X$. Нетрудно видеть, что множества собственных чисел изоморфных потоков совпадают.

Говорят, что поток $R^{t}$ имеет непрерывный спектр, если он не имеет ненулевых собственных значений, и говорят, что $R^{t}$ имеет дискретный спектр, если множество его собственных функций является базисом в $L^{2}(X)$.

Ясно, например, что линейньй поток $R_{\alpha, 1}^{t}$ на торе $\mathbb{T}^{d}$ имеет дискретньй спектр и $\left\{k \alpha+s: k \in \mathbb{Z}^{d}, s \in \mathbb{Z}\right\}$ есть множество его собственных значений. 
Пусть $S$ - автоморфизм пространства Лебега $(X, \mu), f$ - положительная интегрируемая функция на $X$. Обозначим через $M$ подмножество прямого произведения $X \times \mathbb{R}$, составленное из точек $(x, u)$, для которых $0 \leqslant u<f(x)$, и положим для $(x, u) \in M$

$$
T_{S, f}^{t}(x, u)=\left\{\begin{array}{l}
(x, u-t) \quad \text { при }-u \leqslant t<-u-f(x), \\
\left(S^{n} x, u+t-\sum_{k=0}^{n-1} f\left(S^{k} x\right)\right) \\
\text { при }-u+\sum_{k=0}^{n-1} f\left(S^{k} x\right) \leqslant t<-u+\sum_{k=0}^{n} f\left(S^{k} x\right), \quad n \in \mathbb{N}, \\
\left(S^{-n} x, u+t-\sum_{k=1}^{n} f\left(S^{-k} x\right)\right) \\
\text { при }-u-\sum_{k=1}^{n} f\left(S^{-k} x\right) \leqslant t<-u-\sum_{k=1}^{n-1} f\left(S^{-k} x\right), \quad n \in \mathbb{N} .
\end{array}\right.
$$

Нормируем меру в $M$, разделив ее на $\int_{X} f d \mu$. Тогда $T^{t}=T_{S, f}^{t}, t \in \mathbb{R},-$ измеримый поток на пространстве Лебега $M$. Такой поток назьвается специальныцм потоком, построенньм по автоморфизму $S$ и функции $f$.

Рассмотрим специальный поток, построенный по сдвигу тора $T_{\alpha}$ и функции $f \in$ $L^{1}\left(\mathbb{T}^{d}\right)$. Для удобства обозначения будем писать $T_{\alpha, f}^{t}$ вместо $T_{T_{\alpha}, f}^{t}$. Известно, что поток $R_{\alpha, F}^{t}$ может быть реализован как специальньй поток $T_{\alpha, f}^{t}$, построенный по сдвигу $T_{\alpha}$ и функции $f(x)=R(F)$, где $R(F)(x)=\int_{0}^{1} F(x+t \alpha, t) d t$ - время возвращения материальной точки $(x, 0)$, движущейся под действием потока $R_{\alpha, F}^{t}$, на “нулевой уровень" ( $\left.\mathbb{T}^{d}, 0\right)$ (см., например, $\left.[17]\right)$.

Приведем известньй критерий собственных значений специального потока (см. [18]).

ЛЕмма (КСФ). Число $\lambda \in \mathbb{R}$ является собственным значением специального потока $T_{\alpha, f}^{t}$ тогда и только тогда, когда уравнение

$$
h\left(T_{\alpha} x\right)=e^{2 \pi i \lambda f(x)} h(x), \quad x \in \mathbb{T}^{d},
$$

имеет измеримое решение $h: \mathbb{T}^{d} \rightarrow S^{1}$.

Следующая лемма фактически доказана в [19].

ЛЕмма (А.Н. Колмогоров). Если функиия $f, \int_{\mathscr{E} d} f(x) d x=1, f(x)>0$, $x \in \mathbb{T}^{d}$, является тривиальныцм коциклом над сдвигом $T_{\alpha}$, то специальныи поток $T_{\alpha, f}^{t}$ изоморфен потоку $T_{\alpha, 1}^{t}$ ( и соответствующая замена переменных явно выражается через решение когомологического уравнения (1)). 
Для заданных функции Юнга $\psi$ и суммируемой функции $\widetilde{I}(x) \in L^{1}\left(\mathbb{T}^{d}\right)$ определим функциональные пространства:

$$
\begin{aligned}
\widetilde{I} \psi(L)\left(\mathbb{T}^{d}\right) & =\left\{f \in \psi(L)\left(\mathbb{T}^{d}\right): f=\widetilde{I} * g, g \in \psi(L)\left(\mathbb{T}^{d}\right)\right\}, \\
\widetilde{I}_{x} \psi(L)\left(\mathbb{T}^{d+1}\right) & =\left\{F \in \psi(L)\left(\mathbb{T}^{d+1}\right): F=\widetilde{I} * G, G \in \psi(L)\left(\mathbb{T}^{d+1}\right)\right\} .
\end{aligned}
$$

Обозначим через $C^{+}\left(\mathbb{T}^{d}\right)$ множество

$$
\left\{F \in C\left(\mathbb{T}^{d}\right): F(z)>0 \forall z, \int_{\mathscr{E} d} F(x) d x=1\right\} .
$$

Лемма 6.1. Пусть $\widetilde{I} \in L^{1}\left(\mathbb{T}^{d}\right), \psi-$ функиия Юнга. Тогда:

1) линейный оператор $R$ непрерьвно действует из $C\left(\mathbb{T}^{d+1}\right)$ в $C\left(\mathbb{T}^{d}\right)$;

2) для всякой $F \in \widetilde{I}_{x} \psi(L)\left(\mathbb{T}^{d+1}\right)$ функиия $R(F)$ принадлежст пространству $\widetilde{I} \psi(L)\left(\mathbb{T}^{d}\right)$

3) для всякой функции $f \in C^{+}\left(\mathbb{T}^{d}\right) \cap \widetilde{I} \psi(L)\left(\mathbb{T}^{d}\right)$ найдется функция $F \in$ $C^{+}\left(\mathbb{T}^{d+1}\right) \cap \widetilde{I}_{x} \psi(L)\left(\mathbb{T}^{d+1}\right)$, для которой $R(F)=f$.

ДокаЗАТЕльство. 1) Пусть $F \in C\left(\mathbb{T}^{d+1}\right)$. Тогда

$$
\begin{aligned}
\|R(F)\|_{C} & =\max _{x \in \mathscr{E} d}\left|\int_{0}^{1} F(x+t \alpha, t) d t\right| \leqslant \max _{x \in \mathscr{E} d} \max _{t \in[0,1]}|F(x+t \alpha, t)| \\
& =\max _{t \in[0,1]} \max _{x \in \mathscr{E} d}|F(x, t)|=\|F\|_{C} .
\end{aligned}
$$

2) Теперь пусть $F(x, y)=\widetilde{I} * G(x, y)$, где $G \in \psi(L)\left(\mathbb{T}^{d+1}\right)$. Имеем

$$
\begin{aligned}
\widetilde{I} * R(G)(z)=\int_{\mathscr{E} d} \widetilde{I}(x) \int_{0}^{1} G(z-x+t \alpha, t) d t d x \\
\quad=\int_{0}^{1} \int_{\mathscr{E} d} \widetilde{I}(x) G(z-x+t \alpha, t) d x d t=\int_{0}^{1}(\widetilde{I} * G)(z+t \alpha, t) d t=R(F)(z) .
\end{aligned}
$$

Применив неравенство Йенсена к функции $\psi$, получим

$$
\psi(|R(G)(z)|) \leqslant \psi\left(\int_{0}^{1}|G(z+t \alpha, t)| d t\right) \leqslant \int_{0}^{1} \psi(|G(z+t \alpha, t)|) d t
$$

и, значит,

$$
\begin{aligned}
\int_{\mathscr{E} d} \psi(|R(G)(z)|) d z \leqslant \int_{\mathscr{E} d} \int_{0}^{1} \psi(|G(z+t \alpha, t)|) d t d z \\
=\int_{0}^{1} \int_{\mathscr{E} d} \psi(|G(z+t \alpha, t)|) d z d t=\int_{0}^{1} \int_{\mathscr{E} d} \psi(|G(z, t)|) d z d t<\infty,
\end{aligned}
$$

следовательно, $R(G) \in \psi(L)\left(\mathbb{T}^{d}\right)$ и $R(F) \in \widetilde{I} \psi(L)\left(\mathbb{T}^{d}\right)$.

3) Пусть $v:[0,1] \rightarrow \mathbb{R}_{+}-$непрерывная неотрицательная функция, $\int_{0}^{1} v(t) d t=1$, $v(t)=0$ при $t \in[0,1 / 3] \cup[2 / 3,1]$. Пусть $f \in C^{+}\left(\mathbb{T}^{d}\right)$ и $f=\widetilde{I} * g$, где $g \in \psi(L)\left(\mathbb{T}^{d}\right)$. 
Следуя работе Д. В. Аносова [20], определим на кубе $\mathscr{E} d \times[0,1]$ функцию $F$ по формуле $F(x, t)=m+v(t)(f(x-t \alpha)-m)$, где $m=\min _{x \in \mathbb{T}^{d}} f(x)$, и продолжим $F$ до (непрерывной) 1 -периодической функции на $\mathbb{R}^{d} \times \mathbb{R}$ (которую также обозначим через $F)$.

Из определения $F$ заключаем, что $F(x, t)>0$ при всех $x$ и $t$,

$$
\begin{aligned}
\int_{\mathscr{E} d+1} F(z) d z & =m\left(1-\int_{0}^{1} v(t) d t\right)+\int_{0}^{1} \int_{\mathbb{T}^{d}} v(t) f(x-t \alpha) d x d t \\
& =\int_{0}^{1} v(t) d t \int_{\mathscr{E} d} f(x) d x=1
\end{aligned}
$$

и

$$
\int_{0}^{1} F(x+t \alpha, t) d t=\int_{0}^{1}(m+v(t)(f(x)-m)) d t=f(x),
$$

т.е. $f=R(F)$.

Положим $G(x, y)=m+v(y)(g(x-y \alpha)-m)$ для $(x, y) \in \mathscr{E}^{d} \times[0,1]$ и продолжим $G$ до 1-периодической функции на $\mathbb{R}^{d} \times \mathbb{R}($ которую также обозначим через $G$ ). Имеем

$$
\begin{aligned}
\widetilde{I} * G(z, t) & =\int_{\mathscr{E} d} \widetilde{I}(z-x)(m+v(t)(g(x-t \alpha)-m)) d x \\
& =m-m v(t)+v(t) \int_{\mathscr{E} d} \widetilde{I}(z-x) g(x-t \alpha) d x \\
& =m-m v(t)+v(t)(\widetilde{I} * g)(z-t \alpha)=F(z, t) .
\end{aligned}
$$

Так как

$$
\begin{gathered}
\psi(|G(z, t)|) \leqslant \frac{1}{2} \psi(2|m-m v(t)|)+\frac{1}{2} \psi(2 v(t)|g(z-t \alpha)|), \\
\psi(2 v(t)|g(z-t \alpha)|) \leqslant \psi\left(2\|v\|_{\infty}|g(z-t \alpha)|\right) \leqslant 4\|v\|_{\infty}^{2} \psi(|g(z-t \alpha)|),
\end{gathered}
$$

TO

$$
\begin{aligned}
\int_{0}^{1} \int_{\mathscr{E} d} \psi(|G(z, t)|) d z d t & \leqslant C_{1}+2\|v\|_{\infty}^{2} \int_{0}^{1} \int_{\mathscr{E} d} \psi(|g(z-t \alpha)|) d z d t \\
& =C_{1}+2\|v\|_{\infty}^{2} \int_{\mathscr{E} d} \psi(|g(z)|) d z<\infty
\end{aligned}
$$

где $C_{1}=\frac{1}{2} \int_{0}^{1} \psi(2 m|1-v(t)|) d t$.

Tеорема 6.1. Пусть вектор $\alpha \in \mathbb{R}^{d} \quad \beta$-диофантов, пространство $Y=$ $I_{d+\beta, x} L \log _{+}^{1 / 2} L\left(\mathbb{T}^{d+1}\right), d \in \mathbb{N}$, или $Y=W_{x}^{1+\beta} L \log _{+}^{1 / 2} L\left(\mathbb{T}^{2}\right)$, если $d=1$.

Тогда для всякой $F \in Y \cap C^{+}\left(\mathbb{T}^{d+1}\right)$ поток $R_{\alpha, F}^{t}$ изоморфен потоку $R_{\alpha, 1}^{t}$.

ДокАЗАТЕЛЬСТВо. Положим $\theta(0)=0$. При $k \neq 0$ положим $\theta(k) \equiv 1$, если $Y=$ $I_{d+\beta, x} L \log _{+}^{1 / 2} L\left(\mathbb{T}^{d+1}\right)$, и $\theta(k)=\exp \left(-\frac{\pi}{2} i r \operatorname{sign} k\right)$, если $Y=W_{x}^{1+\beta} L \log _{+}^{1 / 2} L\left(\mathbb{T}^{2}\right)$. 
В силу леммы 6.1 для всякой $F \in Y \cap C^{+}\left(\mathbb{T}^{d+1}\right)$ имеем

$$
R(F) \in I_{d+\beta}^{\theta} L \log _{+}^{1 / 2} L\left(\mathbb{T}^{d}\right),
$$

и, значит, в силу теоремы 5.1 функция $R(F)$ есть тривиальный аддитивный коцикл над $T_{\alpha}$. Так как поток $R_{\alpha, F}^{t}$ изоморфен специальному потоку $T_{\alpha, R(F)}^{t}$, то для завершения доказательства осталось использовать лемму Колмогорова.

Обозначим $S^{1}=\{z \in \mathbb{C}:|z|=1\}$.

Лемма 6.2. Пусть $\alpha \in \mathbb{R}^{d}$ - существенно иррациональный вектор. Предположим, что для суммируемой функиии $f: \mathbb{T}^{d} \rightarrow \mathbb{R}$ функциональное уравненue

$$
h(x+\alpha)=e^{2 \pi i f(x)} h(x)
$$

имеет измеримое решение $h: \mathbb{T}^{d} \rightarrow S^{1}$. Тогда последовательность биркгофовых сумм $\left\{\mathbf{B}_{m_{k}}^{\alpha} f\right\}_{k=1}^{\infty}$ сходится по мере для любой $\alpha$-допустимой последовательности $\left\{m_{k}\right\}_{k=1}^{\infty}$.

ДокаЗАтЕльство. Пусть измеримая функция $h: \mathbb{T}^{d} \rightarrow S^{1}$ удовлетворяет (49). Для заданного $\varepsilon>0$ найдем $\delta>0$ такое, что $\omega_{1}(h, \delta)<\left(2 \sin ^{2} \pi \varepsilon\right) / \pi$. (Здесь $\omega_{1}(h, \delta)=\sup _{|y| \leqslant \delta} \int_{\mathscr{E} d}|h(x+y)-h(x)| d x$ - интегральный модуль непрерывности функции $h$.$) Из критерия Кошш сходимости последовательности в полном метри-$ ческом пространстве заключаем, что существует $N \in \mathbb{N}$ такое, что

$$
\max _{s=1, \ldots, d}\left|\left\{m_{j} \alpha_{s}\right\}-\left\{m_{l} \alpha_{s}\right\}\right|<\delta
$$

при всех $j \geqslant N, l \geqslant N$.

Имеем соотношения

$$
\begin{aligned}
\operatorname{mes}\{x & \left.:\left|\mathbf{B}_{m_{j}}^{\alpha} f(x)-\mathbf{B}_{m_{l}}^{\alpha} f(x)\right|>\varepsilon\right\} \leqslant \frac{1}{\sin \pi \varepsilon} \int_{\mathscr{E} d}\left|\sin \pi\left(\mathbf{B}_{m_{j}}^{\alpha} f(x)-\mathbf{B}_{m_{l}}^{\alpha} f(x)\right)\right| d x \\
& =\frac{1}{2 \sin \pi \varepsilon} \int_{\mathscr{E} d}\left|e^{\pi i\left(\mathbf{B}_{m_{j}}^{\alpha} f(x)-\mathbf{B}_{m_{l}}^{\alpha} f(x)\right)}-e^{-\pi i\left(\mathbf{B}_{m_{j}} f(x)-\mathbf{B}_{m_{l}} f(x)\right)}\right| d x \\
& =\frac{1}{2 \sin \pi \varepsilon} \int_{\mathscr{E} d}\left|e^{2 \pi i\left(\mathbf{B}_{m_{j}} f(x)-\mathbf{B}_{m_{l}} f(x)\right)}-1\right| d x \\
& =\frac{1}{2 \sin \pi \varepsilon} \int_{\mathscr{E} d}\left|h\left(x+m_{j} \alpha\right)-h\left(x+m_{l} \alpha\right)\right| d x \\
& =\frac{1}{2 \sin \pi \varepsilon} \int_{\mathscr{E} d}\left|h\left(x+\left\{m_{j} \alpha\right\}\right)-h\left(x+\left\{m_{l} \alpha\right\}\right)\right| d x \\
& =\frac{1}{2 \sin \pi \varepsilon} \int_{\mathscr{E} d}\left|h\left(x+\left\{m_{j} \alpha\right\}-\left\{m_{l} \alpha\right\}\right)-h(x)\right| d x \\
& \leqslant \frac{1}{2 \sin \pi \varepsilon} \omega_{1}(h, \delta)<\frac{\sin \pi \varepsilon}{\pi}<\varepsilon .
\end{aligned}
$$

Следовательно, последовательность $\mathbf{B}_{m_{k}}^{\alpha} f$ фундаментальная (в пространстве $\left.\left(L^{0}\left(\mathbb{T}^{d}\right), \rho_{0}\right)\right)$ и в силу критерия Коши сходится по мере.

Для произвольного подмножества $M$ пространства $L^{1}\left(\mathbb{T}^{d}\right)$ обозначим через $M+1$ множество $\{f(x)=g(x)+1: g \in M\}$. 
ТЕОРема 6.2. Пусть вектор $\alpha \in \mathbb{R}^{d}$ строго $\beta$-диофантов и справедливо любое из следующих четырех условий:

1. $Y=I_{d+\beta, x} L^{1}\left(\mathbb{T}^{d+1}\right)_{0}, \beta>0, d \in \mathbb{N}$;

2. $Y=W_{x}^{1+\beta} L^{1}\left(\mathbb{T}^{2}\right)_{0}, \beta>0, d=1$;

3. $Y=I_{d, x}^{\chi} \psi(L)\left(\mathbb{T}^{d+1}\right)_{0}, \beta=0, d \in \mathbb{N}, \psi \in \Psi, \psi(y)=o\left(y \ln ^{1 / 2} y\right), y \rightarrow+\infty$;

4. $Y=W_{x}^{1} \psi(L)\left(\mathbb{T}^{2}\right)_{0}, \beta=0, d=1, \psi \in \Psi, \psi(y)=o\left(y \ln ^{1 / 2} y\right), y \rightarrow+\infty$.

Тогда для типичной функиии $F$ из метрического пространства

$$
\left(Y \cap C^{+}\left(\mathbb{T}^{d+1}\right), \rho_{C}\right)
$$

число $\lambda=1$ не является собственным значением потока $R_{\alpha, F}^{t}$ (и, значит, поток $R_{\alpha, F}^{t}$ не изоморфен потоку $\left.R_{\alpha, 1}^{t}\right)$.

ДоКАЗАТЕЛЬСТВО. Положим $\theta(0)=0$. При $k \neq 0$ положим $\theta(k) \equiv 1$, если $Y=I_{d+\beta, x} L^{1}\left(\mathbb{T}^{d+1}\right)_{0}, \theta(k)=\exp \left(-\frac{\pi}{2} i r \operatorname{sign} k\right)$, если $Y=W_{x}^{1+\beta} L^{1}\left(\mathbb{T}^{2}\right)_{0}$ или $Y=$ $W_{x}^{1} \psi(L)\left(\mathbb{T}^{2}\right)_{0}$, и $\theta(k)=\chi(k)=\exp \left(2 \pi i|k|_{2} \ln \left(1+|k|_{2}\right)\right)$, если $Y=I_{d, x}^{\chi} \psi(L)\left(\mathbb{T}^{d+1}\right)_{0}$.

Положим $X:=I_{d+\beta}^{\theta} \psi(L)\left(\mathbb{T}^{d}\right)_{0}$. Согласно лемме 4.3 и теореме 4.3 существуют $\alpha$-допустимая последовательность $\left\{m_{j}\right\}_{j=1}^{\infty}$ и множество $V$, удовлетворяющие следующим свойствам:

1 ) множество $V$ есть непустое $G_{\delta}$-подмножество $\left(X, \rho_{0}\right)$;

2) если $f_{0} \in V$, то $\left\{f_{0}+p: p \in \mathscr{T}_{0}^{d}\right\} \subset V$;

3 ) для каждого $f_{0} \in V$ биркгофовы суммы $\mathbf{B}_{m_{j}}^{\alpha}(f), j=1,2, \ldots$, расходятся по мере.

Из теорем вложения $\S 2$ заключаем, что любая функция из пространства $X$ совпадает почти всюду с непрерывной функцией. Так как метрика $\rho_{0}$ не различает совпадающие почти всюду функции, то мы можем считать, что $X \subset C\left(\mathbb{T}^{d}\right)$. При этом ясно, что любое $G_{\delta}$-подмножество пространства $\left(X, \rho_{0}\right)$ является также $G_{\delta}$-подмножеством пространства $\left(X, \rho_{C}\right)$.

Из 1$), 2)$ следует, что множество $V+1$ есть $G_{\delta}$-подмножество пространства $\left(X+1, \rho_{C}\right)$ и что $V+1 \supset\left\{f_{0}(x)+p(x): p \in \mathscr{T}_{0}^{d}\right\}$ для всякой $f_{0} \in V+1$.

По лемме 4.1 оператор $R$ непрерывно действует из $C\left(\mathbb{T}^{d+1}\right)$ в $C\left(\mathbb{T}^{d}\right)$. Поэтому $R^{-1}(V+1)$ есть $G_{\delta}$-подмножество $\left(Y+1, \rho_{C}\right)$.

Пусть $p(x, y)=\sum_{k \in \mathbb{Z}^{d}, l \in \mathbb{Z}} a_{k, l} e(k x+l y) \in \mathscr{T}_{0}^{d+1}$. Тогда

$$
\begin{aligned}
R(p)(x) & =\int_{0}^{1} \sum_{k, l} a_{k, l} e(k(x+y \alpha)+l y) d y=\sum_{k, l} a_{k, l} e(k x) \int_{0}^{1} e(y k \alpha+l y) d y \\
& =\sum_{k}\left(\sum_{l} a_{k, l} \int_{0}^{1} e(y k \alpha+l y) d y\right) e_{k}(x) \in \mathscr{T}_{0}^{d} .
\end{aligned}
$$

Поэтому если $f_{0} \in V+1, F_{0} \in R^{-1}\left(f_{0}\right)$ и $p \in \mathscr{T}_{0}^{d+1}$, то $R\left(F_{0}+p\right)=R\left(F_{0}\right)+$ $R(p) \in V+1$. И значит, множество $R^{-1}(V+1)$ всюду плотно в $\left(Y+1, \rho_{C}\right)$.

Отсюда следует, что $R^{-1}(V+1) \cap C^{+}\left(\mathbb{T}^{d+1}\right)$ - всюду плотное $G_{\delta}$-подмножество $\left(Y_{1} \cap C^{+}\left(\mathbb{T}^{d+1}\right), \rho_{C}\right)$. Для завершения доказательства осталось воспользоваться леммой 6.2 и леммой КСФ.

Полезным дополнением к теореме 6.2 может служить следующая теорема, принадлежашая Б. Файаду [21]. 
Теорема (Б. Файад). Пусть существенно иррациональный вектор $\alpha \in \mathbb{R}^{d}$ не является $\beta$-диофантовым, $\beta>0$. Тогда для типичной функиии $F$ из пространства $\left(C^{\beta+d}\left(\mathbb{T}^{d+1}\right) \cap C^{+}\left(\mathbb{T}^{d+1}\right), \rho_{C}\right)$ поток $R_{\alpha, F}^{t}$ имеет непрерывныи спектр.

ТЕОРема 6.3. 1) Пусть вектор $\alpha \in \mathbb{R}^{d}$ и пространство Х удовлетворяют условиям теоремы 5.1. Тогда для всякой функиии $f \in X, \int_{\mathscr{E} d} f(x) d x=1$, $f(x)>0, x \in \mathbb{T}^{d}$, специальный поток $T_{\alpha, f}^{t}$ изоморфен специальному пото$\kappa y T_{\alpha, 1}^{t}$.

2) Пусть вектор $\alpha \in \mathbb{R}^{d}$ и пространство $X$ удовлетворяют условиям теоремы 5.2. Тогда для типичной функиии $f$ из пространства $((X+1) \cap$ $\left.\left\{f: f(x)>0, x \in \mathbb{T}^{d}\right\}, \rho_{0}\right)$ специальный поток $T_{\alpha, f}^{t}$ не изоморфен потоку $T_{\alpha, 1}^{t}$.

ДокАЗАТЕльство. Пункт 1) теоремы следует из теоремы 5.1 и леммы Колмогорова. Пункт 2) теоремы получаем, применяя лемму 6.2, лемму КСФ и теорему 4.3 так, как это сделано при доказательстве теоремы 6.2 .

В следуюшей теореме Степина [22] проблема изоморфизма специальных потоков исследуется с другой позиции.

Tеорема (А.М. Степин). Пусть $f \in C^{3 / 2+\varepsilon}(\mathbb{T}), \varepsilon>0, \int_{\mathscr{E} d} f(x) d x=1$, $f(x)>0, x \in \mathbb{T}^{d}, u f$ не является тригонометрическим многочленом. Тогда найдется всюду плотное на $[0,1]$ подмнохество $M$ типа $G_{\delta}$ такое, что для $\alpha \in M$ специальные потоки $T_{\alpha, f}^{t}$ и $T_{\alpha, 1}^{t}$ не изоморфны.

\section{§7. О свойствах слабого перемешивания и топологической транзитивности косых с,двигов тора и цилиндра}

Мультипликативным коциклом на $\mathbb{T}^{d}$ назовем измеримое отображение из $\mathbb{T}^{d}$ в $S^{1}=\{z \in \mathbb{C}:|z|=1\}$ - единичную окружность.

Мультипликативный коцикл $\varphi$ называется тривиальным над $T_{\alpha}$, если для некоторой константы $\varkappa \in S^{1}$ и мультипликативного коцикла $h$ справедливо тождество $h(x) \varphi(x)=\varkappa h\left(T_{\alpha} x\right)$.

Мультипликативный коцикл $\varphi$ называется слабо перемешивающим над $T_{\alpha}$, если ни для какого $n \in \mathbb{Z} \backslash\{0\}$ коцикл $\varphi^{n}$ не является тривиальньм над $T_{\alpha}$.

Легко видеть, что если $f$ - тривиальный аддитивный коцикл над $T_{\alpha}$, то $\varphi(x)=$ $e^{2 \pi i f(x)}-$ тривиальный над $T_{\alpha}$ мультипликативњый коцикл.

Заметим также, что каждый мультипликативньй коцикл $\varphi$ может быть представлен в виде $\varphi(x)=e^{2 \pi i f(x)}$, где $f: \mathbb{T}^{d} \rightarrow \mathbb{R}$ - ограниченная измеримая действительнозначная функция. Пусть $M$ - некоторое линейное подпространство $L^{\infty}\left(\mathbb{T}^{d} ; \mathbb{R}\right)$ - пространства измеримых существенно ограниченных действительнозначных функций на $\mathbb{T}^{d}$.

Обозначим через $\Phi(M)$ множество мультипликативных коциклов вида $\varphi(x)=$ $e^{2 \pi i f(x)}$, где $f \in M$. Множество $\Phi(M)$ снабдим групповой операцией поточечного умножения функций и метрикой $\rho_{2}$. Для простоты обозначения вместо $\Phi\left(L^{\infty}\left(\mathbb{T}^{d} ; \mathbb{R}\right)\right)$ будем писать $\Phi$. 
TЕОРема 7.1. Пусть вектор $\alpha \in \mathbb{R}^{d}$ строго $\beta$-диофантов и имеет место любое из следующих четырех условий:

1) $Y=\Phi\left(I_{d+\beta} L^{1}\left(\mathbb{T}^{d}\right)\right), \beta>0, d \in \mathbb{N}$;

2) $Y=\Phi\left(W_{1}^{1+\beta}(\mathbb{T})\right), \beta>0, d=1$;

3) $Y=\Phi\left(I_{d} \psi(L)\left(\mathbb{T}^{d}\right)\right), \beta=0, d \in \mathbb{N}, \psi \in \Psi, \psi(y)=o\left(y \ln ^{1 / 2} y\right), y \rightarrow+\infty$;

4) $Y=\Phi\left(W^{1} \psi(L)(\mathbb{T})\right), \beta=0, d=1, \psi \in \Psi, \psi(y)=o\left(y \ln ^{1 / 2} y\right), y \rightarrow+\infty$.

Тогда слабо перемешивающие над $T_{\alpha}$ мультипликативные коциклы образуют в $\left(Y, \rho_{2}\right)$ всюду плотное множество типа $G_{\delta}$.

ДокАЗАТЕльСТво. Определим функцию $\theta \in \Theta$ как в теореме 5.2 и будем полагать ниже $\varphi(y)=y$ в случае, когда $\beta>0$.

Докажем сначала, что совокупность $\Xi$ коциклов, не являюшихся слабо перемешивающими, образует в $\left(\Phi, \rho_{2}\right)$ множество типа $F_{\sigma}$. Следуя [23], положим

$$
\Phi_{j, k}^{n}:=\left\{\varphi \in \Phi: \exists c \in S^{1}, \exists h \in \Phi \varphi^{n}(x)=c \cdot h\left(T_{\alpha} x\right) \bar{h}(x),|\widehat{\varphi}(j)| \geqslant \frac{1}{k}\right\} .
$$

Нетрудно видеть, что $\Xi=\bigcup_{j \in \mathbb{Z}^{d}} \bigcup_{k \in \mathbb{N}} \bigcup_{n \in \mathbb{Z} \backslash\{0\}} \Phi_{j, k}^{n}$. Докажем, что $\Phi_{j, k}^{n}$ - замкнутое подмножество $\left(\Phi, \rho_{2}\right)$.

Пусть $\varphi_{s} \in \Phi_{j, k}^{n}, s=1,2, \ldots$, и $\varphi_{s}$ сходятся в $\Phi$ к $\varphi_{0}$. Определим $h_{s} \in \Phi$ и $c_{s} \in S^{1}$ из уравнения

$$
\varphi_{s}^{n}(x)=c_{s} \cdot h_{s}\left(T_{\alpha} x\right) \bar{h}_{s}(x) .
$$

В силу слабой компактности единичного шара в $L^{2}\left(\mathbb{T}^{d}\right)$ сушествуют подпоследовательность $\left\{h_{s_{k}}\right\}$ последовательности $\left\{h_{s}\right\}$ и функция $h_{0} \in L^{2}\left(\mathbb{T}^{d}\right)$ такие, что

$$
\int_{\mathscr{E} d} h_{s_{k}}(x) \bar{\xi}(x) d x \rightarrow \int_{\mathscr{E}^{d}} h_{0}(x) \bar{\xi}(x) d x
$$

для любой $\xi \in L^{2}\left(\mathbb{T}^{d}\right)$. Переходя при необходимости к подпоследовательности, будем полагать, что $c_{s_{k}}$ сходятся к некоторому $c_{0} \in S^{1}$. Для краткости записи будем обозначать $h_{s_{k}}$ через $h_{s}$, а $c_{s_{k}}$ через $c_{s}$.

Из слабой сходимости $h_{s}$ к $h_{0}$ следует, что $\widehat{h}_{s}(j) \rightarrow \widehat{h}_{0}(j), s \rightarrow \infty$, и, значит, $\left|\widehat{h}_{0}(j)\right| \geqslant 1 / k$.

Пусть $\xi \in L^{2}\left(\mathbb{T}^{d}\right)$. Имеем

$$
\begin{aligned}
& \left|\int_{\mathscr{E} d} \varphi_{s}^{n}(x) h_{s}(x) \bar{\xi}(x) d x-\int_{\mathscr{E} d} \varphi_{0}^{n}(x) h_{0}(x) \bar{\xi}(x) d x\right| \\
& \quad \leqslant\left|\int_{\mathscr{E} d}\left(\varphi_{s}^{n}(x)-\varphi_{0}^{n}(x)\right) h_{s}(x) \bar{\xi}(x) d x\right|+\left|\int_{\mathscr{E} d} \varphi_{0}^{n}(x)\left(h_{s}(x)-h_{0}(x)\right) \bar{\xi}(x) d x\right| .
\end{aligned}
$$

Из слабой сходимости $h_{s}$ к $h_{0}$ следует, что $\int_{\mathscr{E} d} \varphi_{0}^{n}(x)\left(h_{s}(x)-h_{0}(x)\right) \bar{\xi}(x) d x \rightarrow 0$ при $s \rightarrow \infty$. Так как $\left\|\varphi_{s}^{n}-\varphi_{0}^{n}\right\|_{2} \leqslant n\left\|\varphi_{s}-\varphi_{0}\right\|_{2}$, то

$$
\left|\int_{\mathscr{E} d}\left(\varphi_{s}^{n}(x)-\varphi_{0}^{n}(x)\right) h_{s}(x) \bar{\xi}(x) d x\right| \leqslant\left\|\varphi_{s}^{n}-\varphi_{0}^{n}\right\|_{2}\left\|h_{s} \xi\right\|_{2} \leqslant n\left\|\varphi_{s}-\varphi_{0}\right\|_{2}\|\xi\|_{2} \rightarrow 0
$$

при $s \rightarrow \infty$. 
Аналогично,

$$
\begin{aligned}
& \left|\int_{\mathscr{E} d}\left(c_{s} h_{s}\left(T_{\alpha} x\right)-c_{0} h_{0}\left(T_{\alpha} x\right)\right) \bar{\xi}(x) d x\right| \\
& \quad \leqslant\left|\int_{\mathscr{E} d}\left(c_{s} h_{s}(x)-c_{0} h_{0}(x)\right) \bar{\xi}\left(T_{-\alpha} x\right) d x\right|+\left|\int_{\mathscr{E} d}\left(c_{0} h_{s}(x)-c_{0} h_{0}(x)\right) \bar{\xi}\left(T_{-\alpha} x\right) d x\right| \\
& \quad \leqslant\left|c_{s}-c_{0}\right| \int_{\mathscr{E} d}|\xi(x)| d x+\left|\int_{\mathscr{E} d}\left(h_{s}(x)-h_{0}(x)\right) \bar{\xi}\left(T_{-\alpha} x\right) d x\right| \rightarrow 0
\end{aligned}
$$

при $s \rightarrow \infty$.

Отсюда, вспоминая, что $\varphi_{s}^{n}(x) h_{s}(x)=c_{s} h_{s}\left(T_{\alpha} x\right)$, заключаем, что $\varphi_{0}^{n}(x) h_{0}(x)=$ $c_{0} h_{0}\left(T_{\alpha} x\right)$ для почти всех $x \in \mathbb{T}^{d}$. Следовательно, $\left|h_{0}(x)\right|=\left|\varphi_{0}^{n}(x) h_{0}(x)\right|=$ $\left|c_{0} h_{0}\left(T_{\alpha} x\right)\right|=\left|h_{0}\left(T_{\alpha} x\right)\right|$ при почти всех $x$. Отсюда и из эргодичности автоморфизма $T_{\alpha}$ вытекает, что функция $\left|h_{0}(x)\right|$ - константа на множестве полной меры. При этом константа отлична от нуля, так как $\widehat{h}_{0}(j) \neq 0$. Полагая $h(x)=h_{0}(x) /\left|h_{0}(x)\right|$ $(\in \Phi)$, имеем

$$
\varphi_{0}^{n}(x)=c_{0} h\left(T_{\alpha} x\right) \bar{h}(x) \text { при почти всех } x
$$

и, значит, $\varphi_{0} \in \Phi_{j, k}^{n}$.

Итак, совокупность слабо перемешивающих над $T_{\alpha}$ коциклов в $\left(\Phi, \rho_{2}\right)$ является подмножеством типа $G_{\delta}$ и, значит, при всяком $\psi \in \Psi$ и $\beta \geqslant 0$ слабо перемешивающие над $T_{\alpha}$ коциклы образуют в $\left(\Phi\left(I_{d+\beta}^{\theta} \psi(L)\left(\mathbb{T}^{d}\right)\right), \rho_{2}\right)$ подмножество типа $G_{\delta}$.

Пусть $\varphi$ - произвольный коцикл из $\Phi\left(I_{d+\beta}^{\theta} \psi(L)\left(\mathbb{T}^{d}\right)\right)$. Нетрудно видеть, что коциклы вида $\varphi(x)=\varphi_{0}(x) e^{2 \pi i p(x)}$, где $p \in \mathscr{T}^{d}$, всюду плотны в $\Phi\left(I_{d+\beta}^{\theta} \psi(L)\left(\mathbb{T}^{d}\right)\right)$. Если при этом коцикл $\varphi$ слабо перемешивающий над $T_{\alpha}$, то всякий коцикл вышеуказанного вида также является слабо перемешивающим (над $\left.T_{\alpha}\right)$. Действительно, предположим противное. Пусть для некоторого $p_{0} \in \mathscr{T}^{d}$ сушествуют $n \neq 0, c \in S^{1}$ и $h \in \Phi$ такие, что $\varphi^{n}(x) e^{2 \pi i n p_{0}(x)}=c h\left(T_{\alpha} x\right) \bar{h}(x)$. Но коцикл $e^{-2 \pi i n p_{0}(x)}$ тривиальный над $T_{\alpha}$. Поэтому сушествуют $c_{1} \in S^{1}$ и $h_{1} \in \Phi$ такие, что $e^{-2 \pi i n p_{0}(x)}=c_{1} h_{1}\left(T_{\alpha} x\right) \bar{h}_{1}(x)$, и, значит,

$$
\varphi^{n}(x)=e^{-2 \pi i n p_{0}(x)} \operatorname{ch}\left(T_{\alpha} x\right) \bar{h}(x)=c c_{1} h\left(T_{\alpha} x\right) h_{1}\left(T_{\alpha} x\right) \bar{h}(x) \bar{h}_{1}(x),
$$

т.е. коцикл $\varphi^{n}(x)$ тривиальный над $T_{\alpha}$. Получили противоречие.

Докажем теперь существование слабо перемешивающего коцикла в пространстве $\Phi\left(I_{d+\beta}^{\theta} \psi(L)\left(\mathbb{T}^{d}\right)\right)$. Из теоремы 5.2 следует, что в условиях теоремы существует нетривиальный над $T_{\alpha}$ аддитивньй коцикл $f_{0} \in I_{d+\beta}^{\theta} \psi(L)\left(\mathbb{T}^{d}\right)_{0}$. Из теоремы Мура-Шмидта (см. [24]) немедленно следует, что сушествует $s \in \mathbb{R}$ такое, что

$$
\varphi_{s}(x)=\exp \left(2 \pi i s f_{0}(x)\right)
$$

- нетривиальный над $T_{\alpha}$ мультипликативный коцикл. Положим

$$
S=\left\{s \in \mathbb{R}: \varphi_{s}(x)=\exp \left(2 \pi i s f_{0}(x)\right)-\text { тривиальный коцикл над } T_{\alpha}\right\} .
$$

В работе [24] также доказано, что аддитивная группа $S$ - борелевское подмножество $\mathbb{R}$. 
Так как всякая собственная борелевская подгруппа $\mathbb{R}$ имеет лебегову меру 0 , то mes $\bigcup_{k \neq 0}\{s: k s \in S(f)\}=0$ и, значит, сушествует такое $s_{0} \in \mathbb{R}$, что $k s_{0} \notin S$ при всех $k \in \mathbb{Z} \backslash\{0\}$.

Последнее свойство, очевидно, означает, что коцикл $\exp \left(2 \pi i s_{0} f_{0}(\cdot)\right)$ слабо перемешивающий над $T_{\alpha}$. Теорема полностью доказана.

Пусть $\varphi$ - мультипликативный коцикл, $f \in C\left(\mathbb{T}^{d}\right)$ - непрерывная функция, а $\alpha \in \mathbb{R}^{d}$ - существенно иррациональный вектор. Косьцм сдвигом Анзаи тора $\mathbb{T}^{d} \times S^{1}$ называется его автоморфизм вида

$$
S_{\alpha, \varphi}(x, w)=\left(T_{\alpha} x, w \varphi(x)\right), \quad x \in \mathbb{T}^{d}, w \in S^{1} .
$$

Косым сдвигом цилиндра $\mathbb{T}^{d} \times \mathbb{R}$ (цилиндрическим каскадом) называется автоморфизм цилиндра вида

$$
S^{\alpha, f}(x, y)=\left(T_{\alpha} x, y+f(x)\right), \quad x \in \mathbb{T}^{d}, \quad y \in \mathbb{R} .
$$

Пусть $H$ - подпространство пространства $L^{2}\left(\mathbb{T}^{d} \times S^{1}\right), Q$ - заданный автоморфизм $\mathbb{T}^{d}$. Скажем, что автоморфизм $Q$ имеет на $H$ непрерывный спектр, если пространство $H$ не содержит отличных от тождественно постоянных собственных функций автоморфизма $Q$.

Положим $H_{0}=\left\{F \in L^{2}\left(\mathbb{T}^{d} \times S^{1}\right): F(x, y)=g(x), g(x) \in L^{2}\left(\mathbb{T}^{d}\right)\right\}$ и обозначим через $H_{0}^{\perp}$ ортогональное дополнение к $H_{0}$ в $L^{2}\left(\mathbb{T}^{d} \times S^{1}\right)$.

ТЕОРема 7.2. Пусть вектор $\alpha \in \mathbb{R}^{d}$ и пространство $Y$ удовлетворяют условиям теоремы 7.1. Тогда для типичного мультипликативного коцикла $\psi$ из пространства $\left(Y, \rho_{2}\right)$ косой сдвиг Анзаи $S_{\alpha, \varphi}$ имеет непрерывный спектр на $H_{0}^{\perp}$.

ДокаЗАТЕЛЬСтво. Из работы [25] следует, что косой сдвиг $S_{\alpha, \varphi}$ имеет непрерывный спектр на $H_{0}^{\perp}$ тогда и только тогда, когда мультипликативный коцикл $\psi$ слабо перемешивающий над $T_{\alpha}$.

Tеорема 7.3. Пусть вектор $\alpha \in \mathbb{R}^{d}$ строго $\beta$-диофантов, $X=I_{d+\beta} C\left(\mathbb{T}^{d}\right)_{0}$, если $d \in \mathbb{N}$, или $X=W^{1+\beta} C(\mathbb{T})_{0}$, если $d=1$.

Тогда для типичной функции $f$ из пространства $\left(X, \rho_{C}\right)$ чилиндрический каскад $S^{\alpha, f}$ топологически транзитивен.

ДокАЗАТЕльство. Согласно критерию Хедлунда [26] топологическая транзитивность цилиндрического каскада $S^{\alpha, f}$, построенного по непрерывной функции $f$ с нулевым средним и сушественно иррациональному вектору $\alpha$, равносильна отсутствию непрерьвного решения когомологического уравнения (1), и, значит, требуемое утверждение вытекает из теоремы 5.3.

Автор признателен Д. В. Аносову, А.Б. Катку, А.В. Кочергину, Н. Г. Мошевитину и А.М. Степину за полезные обсуждения.

\section{Список литературы}

1. Кириллов A. А. Динамические системы, факторы и представления грушп // УМН. 1967. T. 22. № 5. C. $67-80$.

2. Степин A. M. О когомологиях групп автоморфизмов пространства Лебега // Функц. анализ и его прилож. 1971. Т. 5. № 2. С. 91-92.

3. Мощевитин Н. Г. О совместных диофонтовых приближениях. Векторы заданного диофантова типа // Матем. заметки. 1997. Т. 61. № 5. С. 706-716. 
4. ШІмидт В. М. Диоффантовы приближения. М.: Мир, 1983.

5. Stein E. M. On limits of sequences of operators // Ann. of Math. 1961. V. 74. P. 140-170.

6. Стейн И. Сингулярные интегралы и дифференциальные свойства функций. М.: Мир, 1973.

7. Зигмунд А. Тригонометрические ряды. Т. 1, 2. М.: Мир, 1965.

8. Красносельский М.А., Рутищкий Я. Б. Вьпуклые функции и пространства Орлича. М.: Физматлит, 1958.

9. Maligranda L., Karlovich A. Yu. On the interpolation constant for Orlicz spaces // Proc. Amer. Math. Soc. V. 129. №9. P. 2727-2739.

10. Wainger $S$. Special trigonometric series in $k$ dimensions // Mem. Amer. Math. Soc. 1965. V. 59.

11. Никольский C. М. Приближение функций многих переменных и теоремы вложения. М.: Наука, 1969.

12. Пичугов C. А. Оценки норм операторов, непрерьвных по мере // Матем. заметки. 1991. T. 50. №1. C. 146-148.

13. Pichugov S. A. Translation invariant operators in linear metric spaces // Anal. Math. 1992. V. 18. № 3. P. 237-248.

14. Рождественский $A$. В. Об абсолютно непрерывных слабо перемешивающих коциклах над иррационалњными поворотами окружности // Матем. сб. 2003. Т. 194. № 5 . C. $139-156$.

15. Стечкин С. Б. Обобщениенекоторых неравенств С.Н. Бернштейна // Докл. АН СССР. 1948. T. 60. № 9. C. 1511-1514.

16. Herman M. R. Sur la conjugaison differentiable des diffeomorphismes du cercle a des rotations // Inst. Hautes Etudes Sci. Publ. Math. 1979. V. 49. P. 5-233.

17. Пуанкаре A. О кривых, определяемых дифференциальньми уравнениями. М.-Л.: ОГИЗ, 1947.

18. Корнфельд И. П., Синай Я. Г., Фомин С. В. Эргодическая теория. М.: Наука, 1980.

19. Колмогоров A. Н. О динамических системах с интегральньм инвариантом на торе // Докл. АН СССР. 1953. Т. 93. С. 763-766.

20. Аносов Д. В. Об аддитивном функциональном гомологическом уравнении, связанном с эргодическим поворотом окружности // Изв. АН СССР. Сер. матем. 1973. Т. 37. №6. C. $1259-1274$.

21. Fayad B. R. Weak mixing for reparametrized linear flows on the torus // Ergodic Theory Dynam. Systems. 2002. V. 22. № 2.

22. Степин A. М. О гомологическом уравнении теории динамических систем // Исследования по теории функций многих вещественных переменных. Тематический межвузовский сборник. Ярославл: Изд-во Ярославского гос. ун-та, 1982. С. 106-117.

23. Iwanik A., Serafin J. Most monothetic extensions are rank-1// Colloq. Math. 1993. V. 56. № 1 . P. $63-76$.

24. Moore C. C., Schmidt K. Coboundaries and homomorphism for nonsingular actions and a problem of H. Helson // Proc. London Math. Soc. (3). 1980. V. 40. P. 443-475.

25. Anzai $H$. Ergodic skew product transformations on the torus // Osaka J. Math. 1951. V. 3. P. 83-99.

26. Gottschalk W.H., Hedlund G.A. Topological dynamics. Providence, RI: Amer. Math. Soc., 1955. (Amer. Math. Soc. Colloq. Publ. V. 36.)

Московский государственнњй университет

Поступила в редакцию

им. М.В. Ломоносова

05.11 .2003

E-mail: arozhd@mech.math.msu.su 